\title{
Time-Space Multiscale Analysis by Use of Tensor Product Wavelets and its Application to Hydrology and GRACE Data
}

\author{
Helga Nutz, Kerstin Wolf \\ Geomathematics Group \\ Department of Mathematics \\ University of Kaiserslautern \\ P.O. Box 3049 \\ 67653 Kaiserslautern \\ Germany \\ Email: hnutz@rhrk.uni-kl.de, kerstin-wlf@gmx.de \\ WWW: http://www.mathematik.uni-kl.de/ wwwgeo
}

June 27, 2007

\begin{abstract}
This paper presents a wavelet analysis of temporal and spatial variations of the Earth's gravitational potential based on tensor product wavelets. The time-space wavelet concept is realized by combining Legendre wavelets for the time domain and spherical wavelets for the space domain. In consequence, a multiresolution analysis for both, temporal and spatial resolution, is formulated within a unified concept. The method is then numerically realized by using first synthetically generated data and, finally, several real data sets.
\end{abstract}

Keywords Multiresolution Analysis, Spherical Wavelets, Legendre Wavelets, Time-Space Multiresolution Analysis.

\section{Introduction}

For our modern industrial society the view from the outer space to the Earth is self-evident. Especially for the investigation of the Earth satellites are an essential tool as they register the inappreciably slow proceeding movement of the crust plates or the smallest changes of the sea level. The direct view into the Earth's interior is provided by a new generation of small satellites which measure the gravitational and magnetic field of the Earth with up to now unknown precision. The remote sensing of the Earth by satellites as, e.g., by the missions for gravity field measurement like CHAMP (CHAllenging Minisatellite Payload), GRACE (Gravity Recovery And Climate Experiment) and GOCE (Gravity field and steady state Ocean Circulation Explorer) provides a considerable and up-to-date data material. In order to get knowledge about the material cycles of oceans, continents and the Earth's interior this information flood has to be evaluated and models about the underlying processes have to be developed. The Earth in change is an interesting and manifold field for science because serious consequences and risks of processes for human life can be estimated. Such regional and global trends can be identified by applying new methods as, e.g., the multiscale analysis presented in this paper which emphasizes the topicality and importance of this research project. 
Since March 2002 the modern twin satellite gravity mission GRACE provides a huge amount of data in form of a complete monthly data set of spherical harmonic coefficients up to order 120. For the first time this enables us to quantify both temporal and spatial variations of the Earth's gravity field caused by mass transport and mass distribution. In the classical approach the gravitational potential is expressed in terms of (scalar) spherical harmonics with time dependent Fourier coefficients.

In this paper a multiresolution using spherical wavelets is applied in order to model spatial variations of the Earth's gravitational potential. In recent years, this method has been developed by the Geomathematics Group of the TU Kaiserslautern, see, e.g., [5] and the references therein. The wavelets are defined in frequency domain which enables us to base our computations directly on the available data. The wavelets serve as band pass filters of Fourier coefficients, where the bandwidth depends on a scale parameter. The higher the scale the higher is the degree of the Fourier coefficients which are filtered out. This is the so-called zooming-in property of the wavelet analysis because with increasing scale finer details are identified. The lower scales represent the coarse parts of the signal whereas the higher scales represent the smaller structures. The advantage of the multiresolution in comparison to the classical approach with spherical harmonics arises from the space localizing property of the used basis functions in contrast to the localization in the frequency domain using spherical harmonics.

The multiresolution method avoids to smear the detail information around the entire globe because the region around the investigated point influences the calculations. This is achieved by describing the signal at each point on the sphere as an adequate combination of frequencies which changes continuously (space evolution of the frequencies). In contrast to this approach the spherical harmonic expansion only localizes in the frequency but not in the spatial domain. The spherical wavelets under use in our approach are radial basis functions on the sphere based on spherical harmonics which filter out certain degrees of the spherical harmonic expansion and, thus, they are an appropriate tool for the analysis of regional characteristics of a signal in $\mathcal{L}^{2}(\Omega)$.

First results concerning the temporal and spatial analysis based on spherical wavelets have been published in [3]. In our paper the temporal wavelet analysis will be realized by introducing the Legendre wavelets which are the counterparts of the spherical wavelets in the space $\mathcal{L}^{2}([-1,1])$. Starting from the temporal and spatial wavelets we are in the position to apply the classical method of tensor product wavelets as introduced, e.g., in [8]. Moreover, we are able to establish pure and hybrid wavelet coefficients which measure both temporal and spatial changes in the gravity signal.

The layout of the paper is as follows: In Section 2 we briefly introduce some important notations and symbols. Section 3 presents a general multiresolution analysis and both the spherical and the Legendre wavelets are introduced. The combined time-space multiresolution analysis for reconstructing a signal in the temporal and spatial domain is discussed in Section 4. Section 5, then, shows a synthetic example and some computations based on GRACE data, see, e.g., [7], (gravititional potential and short time mass variations) and hydrological data from the WGHM (WaterGAP Global Hydrology Model), see, e.g., [2]. In the last Section we summarize our results. 


\section{Preliminaries}

In this section we briefly recapitulate some notation and symbols which will be important within this paper. Further information can be found in [5],[10] and the references therein.

The letters $\mathbb{N}, \mathbb{N}_{0}, \mathbb{Z}$ and $\mathbb{R}$ denote the sets of positive integers, non-negative integers, integers and real numbers, respectively. $\Omega$ is the unit sphere and $\xi, \eta$ represent elements of $\Omega$. The space of all real, square-integrable functions $F$ on $\Omega$ is called $\mathcal{L}^{2}(\Omega) . \mathcal{L}^{2}(\Omega)$ is a Hilbert space with the inner product given by

$$
(F, G)_{\mathcal{L}^{2}(\Omega)}=\int_{\Omega} F(\xi) G(\xi) d \omega(\xi), \quad F, G \in \mathcal{L}^{2}(\Omega) .
$$

In order to introduce an orthonormal basis of $\mathcal{L}^{2}(\Omega)$ we define the scalar spherical harmonics $Y_{n}$ : $\xi \mapsto Y_{n}(\xi)$ of degree $n \in \mathbb{N}_{0}$ as the everywhere on $\Omega$ infinitely often differentiable eigenfunctions of the Beltrami operator $\Delta^{*}$ corresponding to the eigenvalues $\left(\Delta^{*}\right)^{\wedge}(n)=-n(n+1)$, i.e.,

$$
\Delta^{*} Y_{n}=-n(n+1) Y_{n}
$$

The space of all spherical harmonics of degree $n$ is of dimension $2 n+1$ and the set of spherical harmonics $Y_{n, k}: \Omega \rightarrow \mathbb{R}$ of degree $n \in \mathbb{N}_{0}$ and order $k=1, \ldots, 2 n+1$ forms an orthonormal basis of $\mathcal{L}^{2}(\Omega)$. Thus, $F$ can be uniquely represented by a Fourier series in $\mathcal{L}^{2}(\Omega)$-sense

$$
F=\sum_{n=0}^{\infty} \sum_{k=1}^{2 n+1} F^{\wedge}(n, k) Y_{n, k},
$$

where the Fourier coefficients $F^{\wedge}(n, k)$ are given by

$$
F^{\wedge}(n, k)=\int_{\Omega} F(\eta) Y_{n, k}(\eta) d \omega(\eta) .
$$

Closely related to the spherical harmonics are the Legendre polynomials $P_{n}$ of degree $n, n \in$ $\mathbb{N}_{0}$, which are uniquely defined as infinitely often differentiable eigenfunctions of the Legendre operator $L_{t}=\left(\frac{d}{d t}\right)\left(1-t^{2}\right)\left(\frac{d}{d t}\right)$ corresponding to the eigenvalues $-n(n+1)$, i.e.,

$$
L_{t} P_{n}(t)=-n(n+1) P_{n}(t), \quad t \in[-1,1],
$$

and satisfying $P_{n}(1)=1$. Considering the space $\mathcal{L}^{2}([-1,1])$ with scalar product

$$
(F, G)_{\mathcal{L}^{2}([-1,1])}=\int_{-1}^{1} F(t) G(t) d t, \quad F, G \in \mathcal{L}^{2}([-1,1]),
$$

the $\mathcal{L}^{2}([-1,1])$-orthonormal Legendre polynomials $P_{n}^{*}:[-1,1] \rightarrow \mathbb{R}$ given by

$$
P_{n}^{*}=\sqrt{\frac{2 n+1}{2}} P_{n}, \quad n \in \mathbb{N}_{0},
$$

form an orthonormal basis in $\mathcal{L}^{2}([-1,1])$. Therefore, every $F \in \mathcal{L}^{2}([-1,1])$ can be described in a series of the form $F=\sum_{n=0}^{\infty} F^{\wedge}(n) P_{n}^{*}$ which is called the Legendre expansion with Legendre coefficients $F^{\wedge}(n)=\left(F, P_{n}^{*}\right) \mathcal{L}^{2}([-1,1])$. The relation between the Legendre polynomial of degree $n$ and the spherical harmonics of degree $n$ is given by the addition theorem

$$
\sum_{k=1}^{2 n+1} Y_{n, k}(\xi) Y_{n, k}(\eta)=\frac{2 n+1}{4 \pi} P_{n}(\xi \cdot \eta), \quad \xi, \eta \in \Omega .
$$




\section{Multiresolution Analysis}

In this section we present an overview of multiresolution analysis following [6] and the references therein. As a matter of fact, most of the functions in geophysics and geodesy are of bounded energy and, thus, we conclude this section with a short introduction to the Hilbert spaces $\mathcal{L}^{2}([-1,1])$ and $\mathcal{L}^{2}(\Omega)$.

Let $\mathcal{H}$ be a real separable Hilbert space over a certain domain $\Sigma \subset \mathbb{R}^{m}$ with inner product $(\cdot, \cdot)_{\mathcal{H}}$. Furthermore, let $\left\{U_{n}^{*}\right\}_{n \in \mathbb{N}_{0}}$ be an orthonormal system which is complete in $\left(\mathcal{H},(\cdot, \cdot)_{\mathcal{H}}\right)$. Then we define the $\mathcal{H}$-product kernel $\Gamma: \Sigma \times \Sigma \rightarrow \mathbb{R}$ by

$$
\Gamma(x, y)=\sum_{n=0}^{\infty} \Gamma^{\wedge}(n) U_{n}^{*}(x) U_{n}^{*}(y), \quad x, y \in \Sigma,
$$

with symbol $\left\{\Gamma^{\wedge}(n)\right\}_{n \in \mathbb{N}_{0}}$. A kernel function $\Gamma: \Sigma \times \Sigma \rightarrow \mathbb{R}$ is called $\mathcal{H}$-admissible if the following two conditions are satisfied:

1. $\sum_{n=0}^{\infty}\left(\Gamma^{\wedge}(n)\right)^{2}<\infty$,
2. $\sum_{n=0}^{\infty}\left(\Gamma^{\wedge}(n) U_{n}^{*}(x)\right)^{2}<\infty, \quad \forall x \in \Sigma$.

The admissibility conditions guarantee that the functions $\Gamma(x, \cdot): \Sigma \rightarrow \mathbb{R}$ and $\Gamma(\cdot, x): \Sigma \rightarrow \mathbb{R}$, $x \in \Sigma$ fixed, are elements of $\mathcal{H}$. Furthermore, they also ensure that the convolution of an admissible kernel function $\Gamma$ and a function $F \in \mathcal{H}$ is again in $\mathcal{H}$, where the convolution is defined as follows: Let $\Gamma: \Sigma \times \Sigma \rightarrow \mathbb{R}$ be an $\mathcal{H}$-admissible kernel function and $F \in \mathcal{H}$. The convolution of $\Gamma$ against $F$ is defined by

$$
(\Gamma * F)(x)=\int_{\Sigma} F(y) \Gamma(x, y) d y=\sum_{n=0}^{\infty} \Gamma^{\wedge}(n) F^{\wedge}(n) U_{n}^{*}(x) .
$$

Obviously, the symbols fulfill

$$
(\Gamma * F)^{\wedge}(n)=\Gamma^{\wedge}(n) F^{\wedge}(n) .
$$

According to our definition an $\mathcal{H}$-admissible kernel function is in $\mathcal{H}$ if one argument is held fixed. Therefore, we are able to expand the definition of the convolution using two $\mathcal{H}$-admissible kernel functions $\Gamma_{1}$ and $\Gamma_{2}$, which leads to

$$
\begin{aligned}
\left(\Gamma_{1} * \Gamma_{2}\right)(x, y) & =\left(\Gamma_{1} * \Gamma_{2}(\cdot, y)\right)(x) \\
& =\left(\Gamma_{1}(x, \cdot), \Gamma_{2}(\cdot, y)\right)_{\mathcal{H}} \\
& =\sum_{n=0}^{\infty} \Gamma_{1}^{\wedge}(n) \Gamma_{2}^{\wedge}(n) U_{n}^{*}(x) U_{n}^{*}(y),
\end{aligned}
$$

where $x, y \in \Sigma$. The sequence $\left\{\left(\Gamma_{1} * \Gamma_{2}\right)^{\wedge}(n)\right\}_{n \in \mathbb{N}_{0}}$ given by

$$
\left(\Gamma_{1} * \Gamma_{2}\right)^{\wedge}(n)=\Gamma_{1}^{\wedge}(n) \Gamma_{2}^{\wedge}(n),
$$

constitutes an $\mathcal{H}$-admissible kernel function $\Gamma_{1} * \Gamma_{2}$. For brevity we write

$$
\Gamma^{(2)}=\Gamma * \Gamma \text {. }
$$


Our next aim is to define $\mathcal{H}$-scaling functions in such a way that we can interpret them as low pass filters for functions in $\mathcal{H}$. To this end we first introduce the dilation and shifting operators in the following way. Let $\left\{\Gamma_{J}\right\}, J \in \mathbb{Z}$, be a countable family of $\mathcal{H}$-admissible kernel functions. Then the dilation operator $D_{k}, k \in \mathbb{Z}$, is defined by

$$
D_{k} \Gamma_{J}=\Gamma_{J+k},
$$

and the shifting operator $S_{x}, x \in \Sigma$, is defined by

$$
S_{x} \Gamma_{J}=\Gamma_{J}(x, \cdot) .
$$

The kernel function $\Gamma_{0} \in\left\{\Gamma_{J}\right\}_{J \in \mathbb{Z}}$ is called the mother kernel of the family and

$$
\Gamma_{J}(x, \cdot)=S_{x} D_{J} \Gamma_{0}
$$

holds for all $x \in \Sigma$ and all $J \in \mathbb{Z}$. Next we introduce the generating symbol of an $\mathcal{H}$-scaling function which finally leads to the definition of the $\mathcal{H}$-scaling function. Let $\left(\Phi_{0}\right)^{\wedge}(n)$ be the symbol of an $\mathcal{H}$-admissible kernel function which additionally satisfies the following two conditions:

1. $\left(\Phi_{0}\right)^{\wedge}(0)=1$,

2. if $n>k$ then $\left(\Phi_{0}\right)^{\wedge}(n) \leq\left(\Phi_{0}\right)^{\wedge}(k)$.

Then $\left(\Phi_{0}\right)^{\wedge}(n)$ is called the generating symbol of the mother $\mathcal{H}$-scaling function given by

$$
\Phi_{0}(x, y)=\sum_{n=0}^{\infty}\left(\Phi_{0}\right)^{\wedge}(n) U_{n}^{*}(x) U_{n}^{*}(y), \quad x, y \in \Sigma .
$$

Since we are interested in the dilated versions of the mother $\mathcal{H}$-scaling function we have to extend the definition of the generating symbol in the following way: Let $\left\{\left(\Phi_{J}\right)^{\wedge}(n)\right\}_{n \in \mathbb{N}_{0}}, J \in \mathbb{Z}$, be an $\mathcal{H}$-admissible symbol satisfying, in addition, the following properties:

1. $\lim _{J \rightarrow \infty}\left(\left(\Phi_{J}\right)^{\wedge}(n)\right)^{2}=1, \quad n \in \mathbb{N}$,

2. $\left(\left(\Phi_{J}\right)^{\wedge}(n)\right)^{2} \geq\left(\left(\Phi_{J-1}\right)^{\wedge}(n)\right)^{2}, \quad J \in \mathbb{Z}, n \in \mathbb{N}$,

3. $\lim _{J \rightarrow-\infty}\left(\left(\Phi_{J}\right)^{\wedge}(n)\right)^{2}=0, \quad n \in \mathbb{N}$,

4. $\left(\left(\Phi_{J}\right)^{\wedge}(0)\right)^{2}=1, \quad J \in \mathbb{Z}$.

Then $\left\{\left(\Phi_{J}\right)^{\wedge}(n)\right\}_{n \in \mathbb{N}_{0}}, J \in \mathbb{Z}$, is called the generating symbol of an $\mathcal{H}$-scaling function. The corresponding family $\left\{\Phi_{J}\right\}_{J \in \mathbb{Z}}$ of kernel functions given by

$$
\Phi_{J}(x, y)=\sum_{n=0}^{\infty}\left(\Phi_{J}\right)^{\wedge}(n) U_{n}^{*}(x) U_{n}^{*}(y), \quad x, y \in \Sigma,
$$

is called $\mathcal{H}$-scaling function. Note that the $\mathcal{H}$-scaling functions can also be introduced by

$$
\Phi_{J}(x, \cdot)=D_{J} S_{x} \Phi_{0}(\cdot, \cdot),
$$

i.e., the $\mathcal{H}$-scaling functions are the dilated and shifted versions of the mother $\mathcal{H}$-scaling function. In order to define the associated $\mathcal{H}$-wavelets we let $\left\{\left(\Phi_{J}\right)^{\wedge}(n)\right\}_{n \in \mathbb{N}_{0}}, J \in \mathbb{Z}$, be the generating symbol of an $\mathcal{H}$-scaling function. Then the generating symbol

$$
\left\{\left(\Psi_{J}\right)^{\wedge}(n)\right\}_{n \in \mathbb{N}_{0}}, \quad J \in \mathbb{Z},
$$


of the associated $\mathcal{H}$-wavelet is defined by the refinement equation

$$
\Psi_{J}^{\wedge}(n)=\sqrt{\left(\Phi_{J+1}^{\wedge}(n)\right)^{2}-\left(\Phi_{J}^{\wedge}(n)\right)^{2}}, \quad n \in \mathbb{N}_{0} .
$$

The family $\left\{\Psi_{J}\right\}_{J \in \mathbb{Z}}$ of $\mathcal{H}$-product kernels given by

$$
\Psi_{J}(x, y)=\sum_{n=0}^{\infty}\left(\Psi_{J}\right)^{\wedge}(n) U_{n}^{*}(x) U_{n}^{*}(y), \quad x, y \in \Sigma,
$$

is called $\mathcal{H}$-wavelet associated to the $\mathcal{H}$-scaling function $\left\{\Phi_{J}\right\}, J \in \mathbb{Z}$. The corresponding mother wavelet is denoted by $\Psi_{0}$. Again we can interpret the $\mathcal{H}$-wavelets as dilated and shifted versions of the corresponding mother wavelet, i.e.,

$$
\Psi_{J}(x, \cdot)=S_{x} D_{J} \Psi_{0}(\cdot, \cdot) .
$$

By virtue of the scaling functions and wavelets we define the scale spaces $\mathcal{V}_{J}$ and the detail spaces $\mathcal{W}_{J}$ by

$$
\mathcal{V}_{J}=\left\{\Phi_{J}^{(2)} * F \mid F \in \mathcal{H}\right\}
$$

and

$$
\mathcal{W}_{J}=\left\{\Psi_{J}^{(2)} * F \mid F \in \mathcal{H}\right\}
$$

The spaces $\mathcal{V}_{J}$ represent the scale approximation of $F$ at scale $J$ and the corresponding operator $T_{J}(F)=\Phi_{J}^{(2)} * F$ can be interpreted as a low pass filter, whereas the spaces $\mathcal{W}_{J}$ represent the wavelet approximation of $F$ at scale $J$ and the corresponding operator $R_{J}(F)=\Psi_{J}^{(2)} * F$ can be interpreted as a band pass filter. We have the decomposition

$$
\mathcal{V}_{J+1}=\mathcal{V}_{J}+\mathcal{W}_{J}
$$

and the limit relation (in $\mathcal{H}$-sense)

$$
\lim _{J \rightarrow \infty} \Phi_{J}^{(2)} * F=F,
$$

which leads to the multiresolution analysis given by the nested sequence of scale spaces

$$
\ldots \subset \mathcal{V}_{J} \subset \mathcal{V}_{J+1} \subset \ldots \subset \mathcal{H}
$$

and

$$
\mathcal{H}=\bigcup_{J=-\infty}^{\infty} \mathcal{V}_{J}^{\|\cdot\|_{\mathcal{H}}}
$$

Therefore, we can decompose the space $\mathcal{V}_{J}$ for each scale $J \in \mathbb{Z}$ in one 'basic' scale space and several detail spaces:

$$
\mathcal{V}_{J}=\mathcal{V}_{J_{0}}+\sum_{j=J_{0}}^{J-1} \mathcal{W}_{j}
$$

The convolution

$$
(W T)(F)(J ; x)=\left(\Psi_{J} * F\right)(x)
$$

is called wavelet coefficient of $F$ at scale $J \in \mathbb{Z}$ and location $x \in \Sigma$. The wavelet coefficients provide the decomposition of $F$, whereas for the reconstruction of $F$ from its wavelet coefficients we need the double convolution $\Psi_{J}^{(2)} * F=\Psi_{J} *(W T)(F)(J ; \cdot)$.

In the following two subsections we will remind the Hilbert spaces $\mathcal{L}^{2}([-1,1])$ used for Legendre wavelets and $\mathcal{L}^{2}(\Omega)$ used for spherical wavelets. 


\subsection{Legendre Wavelets}

We will now consider $\mathcal{H}=\mathcal{L}^{2}([-1,1])$, the space of square-integrable functions $F:[-1,1] \rightarrow \mathbb{R}$, i.e., we let $\Sigma=[-1,1]$. This choice leads to the so-called Legendre wavelets (cf. [1]). We already defined the inner product $(F, G)_{\mathcal{L}^{2}([-1,1])}$ and the orthonormal system of Legendre polynomials $P_{n}^{*}$. The $\mathcal{L}^{2}([-1,1])$-admissible product kernels, then, are given by

$$
\Gamma(s, t)=\sum_{n=0}^{\infty} \Gamma^{\wedge}(n) P_{n}^{*}(s) P_{n}^{*}(t), \quad s, t \in[-1,1],
$$

and the convolution of $\Gamma$ against $F$ is given by

$$
(\Gamma * F)(t)=\sum_{n=0}^{\infty} \Gamma^{\wedge}(n) F^{\wedge}(n) P_{n}^{*}(t), \quad t \in[-1,1] .
$$

\subsection{Spherical Wavelets}

In case of the scalar spherical wavelet theory we let $\Sigma=\Omega$ and consider $\mathcal{H}=\mathcal{L}^{2}(\Omega)$. As an $\mathcal{L}^{2}(\Omega)$-orthonormal system we choose the system $\left\{Y_{n, k}\right\}_{n \in \mathbb{N}_{0}, k=1, \ldots, 2 n+1}$ of spherical harmonics of degree $n$ and order $k$. The $\mathcal{L}^{2}(\Omega)$-product kernels have the following representation

$$
\Gamma(\xi, \eta)=\sum_{n=0}^{\infty} \sum_{k=1}^{2 n+1} \Gamma^{\wedge}(n) Y_{n, k}(\xi) Y_{n, k}(\eta), \quad \xi, \eta \in \Omega,
$$

whereas the convolution of $\Gamma$ against $F$ is given by

$$
(\Gamma * F)(\xi)=\sum_{n=0}^{\infty} \sum_{k=1}^{2 n+1} \Gamma^{\wedge}(n) F^{\wedge}(n, k) Y_{n, k}(\xi), \quad \xi \in \Omega .
$$

\section{Time-Space Multiresolution Analysis}

We will now combine the Legendre wavelet expansion for the temporal analysis with the spherical wavelet expansion for the analysis of spatial variations. This is done by introducing timespace dependent tensor product wavelets. Further information about the time-space dependent wavelet theory can be found in, e.g., [4], [9] and the references therein. Starting point of our considerations is the space $\mathcal{L}^{2}([-1,1] \times \Omega)$ where without loss of generality we assume the time interval to be normalized to the interval $[-1,1]$. The scalar product of $F, G \in \mathcal{L}^{2}([-1,1] \times \Omega)$ is given by

$$
(F, G)_{\mathcal{L}^{2}([-1,1] \times \Omega)}=\int_{-1}^{1} \int_{\Omega} F(t ; \xi) G(t ; \xi) d \omega(\xi) d t .
$$

We assume that the time dependency is fully described by the spatial Fourier coefficients, i.e.,

$$
F(t ; \xi)=\sum_{n=0}^{\infty} \sum_{k=1}^{2 n+1} F^{\wedge}(n, k)(t) Y_{n, k}(\xi),
$$

with

$$
F^{\wedge}(n, k)(t)=\sum_{n^{\prime}=0}^{\infty} F^{\wedge}\left(n^{\prime} ; n, k\right) P_{n^{\prime}}^{*}(t) .
$$


Note that, for notational reasons, in the following $n^{\prime}$ will always be used in case of time, whereas $n$ will be used in space. We finally arrive at

$$
F=\sum_{n^{\prime}=0}^{\infty} \sum_{n=0}^{\infty} \sum_{k=1}^{2 n+1} F^{\wedge}\left(n^{\prime} ; n, k\right) P_{n^{\prime}}^{*} Y_{n, k}
$$

in $\mathcal{L}^{2}([-1,1] \times \Omega)$-sense.

Our aim is to define the scaling functions and wavelets in such a way that we result in a multiresolution of $\mathcal{L}^{2}([-1,1] \times \Omega)$ of the form

$$
\ldots \subset \mathcal{V}_{J} \subset \mathcal{V}_{J+1} \subset \ldots \subset \mathcal{L}^{2}([-1,1] \times \Omega)
$$

and

$$
\mathcal{L}^{2}([-1,1] \times \Omega)=\overline{\bigcup_{J=-\infty}^{\infty} \mathcal{V}_{J}} \|_{\mathcal{L}^{2}([-1,1] \times \Omega)}
$$

In the following section we use results of higher dimensional multiresolution analysis as presented in [8] and some ideas described in [9] for the extension to the space $\mathcal{L}^{2}([-1,1] \times \Omega)$. We start with the definition of the generating symbol of time-space scaling functions. Let $\left\{\left(\Phi^{\prime}{ }_{J}\right)^{\wedge}\left(n^{\prime}\right)\right\}_{n^{\prime} \in \mathbb{N}_{0}}$, $J \in \mathbb{Z}$, be the generating symbol of a temporal scaling function and $\left\{\left(\Phi_{J}\right)^{\wedge}(n)\right\}_{n \in \mathbb{N}_{0}}, J \in \mathbb{Z}$, be the generating symbol of a spatial scaling function. Then the generating symbol of the time-space (tensor product) scaling function is given by the sequence

$$
\left\{\left(\tilde{\Phi}_{J}\right)^{\wedge}\left(n^{\prime} ; n\right)\right\}_{n^{\prime}, n \in \mathbb{N}_{0}},
$$

with

$$
\left(\tilde{\Phi}_{J}\right)^{\wedge}\left(n^{\prime} ; n\right)=\left(\Phi^{\prime}{ }_{J}\right)^{\wedge}\left(n^{\prime}\right)\left(\Phi_{J}\right)^{\wedge}(n) .
$$

Now we are able to define the time-space scaling function. Let $\left\{\left(\tilde{\Phi}_{J}\right)^{\wedge}\left(n^{\prime} ; n\right)\right\}_{n^{\prime}, n \in \mathbb{N}_{0}}, J \in \mathbb{Z}$, be the symbol of a time-space scaling function. Then the family of kernel functions $\left\{\tilde{\Phi}_{J}\right\}_{J \in \mathbb{Z}}$ defined by

$$
\tilde{\Phi}_{J}(s, t ; \xi, \eta)=\sum_{n^{\prime}=0}^{\infty} \sum_{n=0}^{\infty} \sum_{k=1}^{2 n+1}\left(\tilde{\Phi}_{J}\right)^{\wedge}\left(n^{\prime} ; n\right) P_{n^{\prime}}^{*}(s) P_{n^{\prime}}^{*}(t) Y_{n, k}(\xi) Y_{n, k}(\eta),
$$

where $s, t \in[-1,1]$ and $\xi, \eta \in \Omega$, denote the time-space (tensor product) scaling functions. Since we have two refinement equations

$$
\begin{gathered}
\left(\left(\Psi^{\prime}{ }_{J}\right)^{\wedge}\left(n^{\prime}\right)\right)^{2}=\left(\left(\Phi^{\prime}{ }_{J+1}\right)^{\wedge}\left(n^{\prime}\right)\right)^{2}-\left(\left(\Phi^{\prime}{ }_{J}\right)^{\wedge}\left(n^{\prime}\right)\right)^{2} \\
\left(\left(\Psi_{J}\right)^{\wedge}(n)\right)^{2}=\left(\left(\Phi_{J+1}\right)^{\wedge}(n)\right)^{2}-\left(\left(\Phi_{J}\right)^{\wedge}(n)\right)^{2} .
\end{gathered}
$$

which have to be fulfilled simultaneously we get

$$
\begin{aligned}
\left(\left(\Phi_{J+1}^{\prime}\right)^{\wedge}\left(n^{\prime}\right)\right)^{2}\left(\left(\Phi_{J+1}\right)^{\wedge}(n)\right)^{2}= & \left(\left(\Phi_{J}^{\prime}\right)^{\wedge}\left(n^{\prime}\right)\right)^{2}\left(\left(\Phi_{J}\right)^{\wedge}(n)\right)^{2} \\
& +\left(\left(\Psi_{J}^{\prime}\right)^{\wedge}\left(n^{\prime}\right)\right)^{2}\left(\left(\Phi_{J}\right)^{\wedge}(n)\right)^{2} \\
& +\left(\left(\Phi^{\prime}{ }_{J}\right)^{\wedge}\left(n^{\prime}\right)\right)^{2}\left(\left(\Psi_{J}\right)^{\wedge}(n)\right)^{2} \\
& +\left(\left(\Psi_{J}^{\prime}\right)^{\wedge}\left(n^{\prime}\right)\right)^{2}\left(\left(\Psi_{J}\right)^{\wedge}(n)\right)^{2} .
\end{aligned}
$$


This leads to the definition of two hybrid wavelets $\tilde{\Psi}_{J}^{1}$ and $\tilde{\Psi}_{J}^{2}$ and one pure wavelet $\tilde{\Psi}_{J}^{3}$ :

$$
\tilde{\Psi}_{J}^{1}(s, t ; \xi, \eta)=\sum_{n^{\prime}=0}^{\infty} \sum_{n=0}^{\infty} \sum_{k=1}^{2 n+1}\left(\tilde{\Psi}_{J}^{1}\right)^{\wedge}\left(n^{\prime} ; n\right) P_{n^{\prime}}^{*}(s) P_{n^{\prime}}^{*}(t) Y_{n, k}(\xi) Y_{n, k}(\eta)
$$

with $\left(\tilde{\Psi}_{J}^{1}\right)^{\wedge}\left(n^{\prime} ; n\right)=\left(\Phi^{\prime}{ }_{J}\right)^{\wedge}\left(n^{\prime}\right)\left(\Psi_{J}\right)^{\wedge}(n)$ and

$$
\tilde{\Psi}_{J}^{2}(s, t ; \xi, \eta)=\sum_{n^{\prime}=0}^{\infty} \sum_{n=0}^{\infty} \sum_{k=1}^{2 n+1}\left(\tilde{\Psi}_{J}^{2}\right)^{\wedge}\left(n^{\prime} ; n\right) P_{n^{\prime}}^{*}(s) P_{n^{\prime}}^{*}(t) Y_{n, k}(\xi) Y_{n, k}(\eta)
$$

with $\left(\tilde{\Psi}_{J}^{2}\right)^{\wedge}\left(n^{\prime} ; n\right)=\left(\Psi_{J}^{\prime}\right)^{\wedge}\left(n^{\prime}\right)\left(\Phi_{J}\right)^{\wedge}(n)$ and

$$
\tilde{\Psi}_{J}^{3}(s, t ; \xi, \eta)=\sum_{n^{\prime}=0}^{\infty} \sum_{n=0}^{\infty} \sum_{k=1}^{2 n+1}\left(\tilde{\Psi}_{J}^{3}\right)^{\wedge}\left(n^{\prime} ; n\right) P_{n^{\prime}}^{*}(s) P_{n^{\prime}}^{*}(t) Y_{n, k}(\xi) Y_{n, k}(\eta)
$$

with $\left(\tilde{\Psi}_{J}^{3}\right)^{\wedge}\left(n^{\prime} ; n\right)=\left(\Psi_{J}^{\prime}\right)^{\wedge}\left(n^{\prime}\right)\left(\Psi_{J}\right)^{\wedge}(n)$.

For deducing the multiresolution we first introduce the time-space convolution. Let $F \in$ $\mathcal{L}^{2}([-1,1] \times \Omega)$ and let $\Gamma$ be a kernel function of the form

$$
\Gamma(s, t ; \xi, \eta)=\sum_{n^{\prime}=0}^{\infty} \sum_{n=0}^{\infty} \sum_{k=1}^{2 n+1} \Gamma^{\wedge}\left(n^{\prime} ; n\right) P_{n^{\prime}}^{*}(s) P_{n^{\prime}}^{*}(t) Y_{n, k}(\xi) Y_{n, k}(\eta) .
$$

The time-space convolution of $\Gamma$ against $F$ is defined by

$$
\begin{aligned}
(\Gamma \star F)(t ; \eta) & =\int_{-1}^{1} \int_{\Omega} \Gamma(s, t ; \xi, \eta) F(s ; \xi) d \omega(\xi) d s \\
& =\sum_{n^{\prime}=0}^{\infty} \sum_{n=0}^{\infty} \sum_{k=1}^{2 n+1} \Gamma^{\wedge}\left(n^{\prime} ; n\right) F^{\wedge}\left(n^{\prime} ; n, k\right) P_{n^{\prime}}^{*}(t) Y_{n, k}(\eta) .
\end{aligned}
$$

The convolution of two kernel functions is defined in analogous manner. Now we introduce the scale and wavelet spaces. Let $\left\{\tilde{\Phi}_{J}\right\}$ be the time-space scaling functions and let $\left\{\tilde{\Psi}_{J}^{1}\right\},\left\{\tilde{\Psi}_{J}^{2}\right\}$ and $\left\{\tilde{\Psi}_{J}^{3}\right\}$ be the associated hybrid and pure time-space wavelets at scale $J$. Then the pure time-space scale spaces are defined by

$$
\tilde{\mathcal{V}}_{J}=\left\{\tilde{\Phi}_{J} \star \tilde{\Phi}_{J} \star F \mid F \in \mathcal{L}^{2}([-1,1] \times \Omega)\right\} .
$$

The hybrid time-space detail spaces are given by

$$
\begin{aligned}
& \tilde{\mathcal{W}}_{J}^{1}=\left\{\tilde{\Psi}_{J}^{1} \star \tilde{\Psi}_{J}^{1} \star F \mid F \in \mathcal{L}^{2}([-1,1] \times \Omega)\right\} \\
& \tilde{\mathcal{W}}_{J}^{2}=\left\{\tilde{\Psi}_{J}^{2} \star \tilde{\Psi}_{J}^{2} \star F \mid F \in \mathcal{L}^{2}([-1,1] \times \Omega)\right\}
\end{aligned}
$$

while the pure time-space detail spaces are defined by

$$
\tilde{\mathcal{W}}_{J}^{3}=\left\{\tilde{\Psi}_{J}^{3} \star \tilde{\Psi}_{J}^{3} \star F \mid F \in \mathcal{L}^{2}([-1,1] \times \Omega)\right\} .
$$

The next theorem which shows that the characteristics of a multiresolution analysis are fulfilled by the scale and detail spaces can be proven similar to [9]. 
Theorem 4.1 For $J \in \mathbb{Z}$ let $\left\{\tilde{\Phi}_{J}\right\}$ be time-space scaling functions and let $\left\{\tilde{\Psi}_{J}^{1}\right\}$, $\left\{\tilde{\Psi}_{J}^{2}\right\}$ and $\left\{\tilde{\Psi}_{J}^{3}\right\}$ be the associated hybrid and pure time-space wavelets. Suppose that $F \in \mathcal{L}^{2}([-1,1] \times \Omega)$. Then

$$
\begin{aligned}
F & =\lim _{J \rightarrow \infty}\left(\tilde{\Phi}_{J}^{(2)} \star F\right) \\
& =\lim _{J \rightarrow \infty}\left(\tilde{\Phi}_{J_{0}}^{(2)} \star F+\sum_{j=J_{0}} \sum_{i=1}^{3}\left(\tilde{\Psi}_{j}^{i}\right)^{(2)} \star F\right)
\end{aligned}
$$

holds true in the sense of the $\mathcal{L}^{2}([-1,1] \times \Omega)$-metric. Accordingly, for the time-space scale spaces and detail spaces we have

$$
\tilde{\mathcal{V}}_{J}=\tilde{\mathcal{V}}_{J_{0}}+\sum_{j=J_{0}}^{J-1} \sum_{i=1}^{3} \tilde{\mathcal{W}}_{j}^{i}
$$

with $J, J_{0} \in \mathbb{Z}$ and $J_{0} \leq J$.

\section{Numercical Results with Synthetic Data and Real Data}

This section is dedicated to the numerical computations with synthetic and real data as application of the time-space multiresolution method explained in the last sections. First, we present some results based on synthetically generated data in order to get an impression of the efficiency of the method. We choose a trial function combining both variations in time and in space. Then some results for real Earth's gravitational data from the satellite mission GRACE, hydrological data (WGHM) and atmospheric short time mass variations (GRACE) are given. The data have been provided from our project partners from GeoForschungsZentrum (GFZ) Potsdam, Department 1: Geodäsie und Fernerkundung within the TIVAGAM-project which is part of the 'Geotechnologies Special Programme' funded by the 'Federal Ministry of Education and Research'.

In case of the Earth's gravity field (GRACE) the data are given as a time series of 40 monthly data sets given from February 2003 to May 2006. The wavelet analysis requires equidistant data in time and for reasons of two missing months (June 2003 and January 2004) we have to complete the time series by linear interpolation. The monthly data sets for the gravity field are given as spherical harmonic expansions complete up to degree and order 120. The atmospheric short time mass variations are given as a time series consisting of 124 dates in March 2003 (from March 1st at 0:00 to March 31st at 18:00) where the data sets are provided every 6 hours. In this case the data are given up to degree and order 100. In case of hydrological data (WGHM) we use 36 monthly data sets from January 2003 to December 2005 which are given as water columns.

We restrict our numerical realization on the so-called cubic polynomial wavelet. The symbol $\left(\tilde{\Phi}_{J}\right)^{\wedge}(n)$ of the corresponding scaling functions in time and space (2) is composed by the temporal and spatial symbols $\left(\Phi_{J}^{\prime}\right)^{\wedge}(n)$ and $\left(\Phi_{J}\right)^{\wedge}(n)$ given by

$$
\left(\Phi^{\prime}{ }_{J}\right)^{\wedge}(n)=\left(\Phi_{J}\right)^{\wedge}(n)= \begin{cases}\left(1-2^{-J} n\right)^{2}\left(1+2^{1-J} n\right) & , 0 \leq n<2^{J}, \\ 0 & , n \geq 2^{J},\end{cases}
$$

for $n, J \in \mathbb{N}_{0}$. Note that for the computations the scale $J$ is non-negative. Figure 1 and Figure 2 show the spatial wavelet symbols and the spatial wavelet functions, respectively, where the wavelet symbols are calculated via the refinement equation (1). 


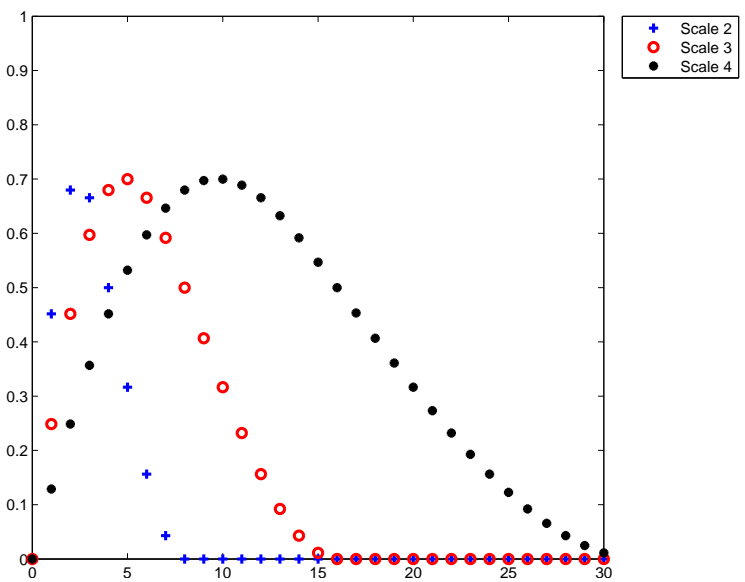

Figure 1: Wavelet symbols $n \mapsto\left(\Psi_{J}\right)^{\wedge}(n)$ for $n=0,1, \ldots, 30$ and $J=2,3,4$.

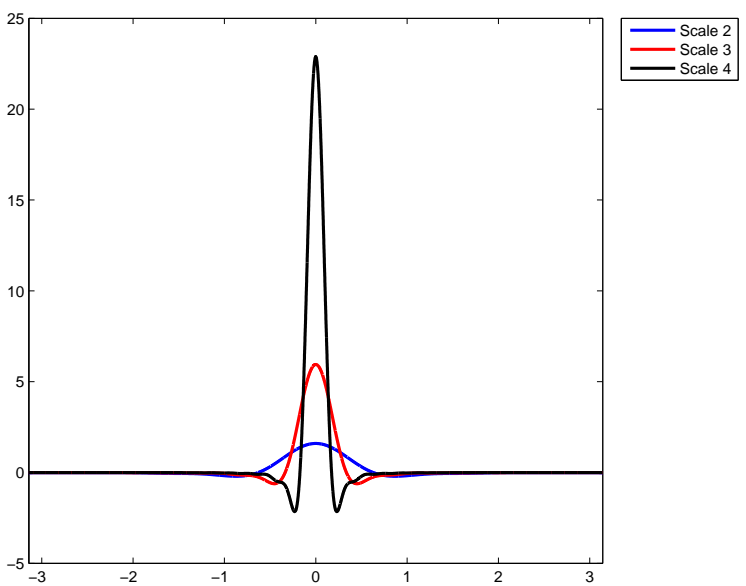

Figure 2: Wavelet functions $\vartheta \mapsto \Psi_{J}(\cos (\vartheta))$ for $\vartheta \in[-\pi, \pi]$ and $J=2,3,4$.

Phenomena which occur both in time and space have to be described using four dimensions, one in time and three in space, which leads to difficulties as far as the presentation of the results is concerned. To extract the important characteristics we decided to use two types of presentation: In order to show the spatial distribution of the detected phenomena we plot the maximum of the absolute values over all wavelet coefficients of the point under consideration. In doing so, we get an idea where the positions of great or moderate changes (over the whole interval) are. Furthermore, we select special positions located in regions of interest and plot the time dependent courses. This helps us to understand seasonal or other temporal variations.

For reasons of comparison between different scales in case of wavelet coefficients we have adapted the color scale of the corresponding plots. The values 'min' and 'max' under these figures give us information about the real minimal and maximal values. The plots of the scaling functions are not adapted in order to make clear the reconstruction of the original signal. Note that for the computations the time interval has to be normalized to $[-1,1]$, but for reasons of understanding we prefer the original marks of the dates in the plots.

\subsection{Synthetically Generated Data}

In order to get a realistic simulation of the situation given in case of real GRACE data we decided to analyze a synthetically generated time series consisting of 40 dates. Starting from a certain trial function $F(t ; \xi), t \in[1,40], \xi \in \Omega$, we calculate the corresponding spherical harmonic coefficients $F^{\wedge}(n, j)(t), n \in \mathbb{N}_{0}, k=1, \ldots, 2 n+1, t \in[1,40]$, to obtain the input data sets. In order to give an impression of the efficiency of the algorithm we use a trial function which contains temporal and spatial variations (a moving smoothed peak).

The trial function depends on time and space and is given by

$$
F(t ; \xi)= \begin{cases}\frac{1}{|\xi-\eta(t)|} & , \text { for all } t \in[10,30], \\ 0 & , \text { for all } t \in[1,9] \cup[31,40],\end{cases}
$$

where $\eta(t)$ moves along the equator. The maximum value of the first smoothed peak, i.e., for $t=10$, is located at $90^{\circ} \mathrm{W} 0^{\circ} \mathrm{N}$ and the last one at $90^{\circ} \mathrm{E} 0^{\circ} \mathrm{N}$, such that we have 21 points 
at intervals of $9^{\circ}$. The maximum values of the trial function over the whole time interval are plotted in Figure 3(a). The time dependent courses are given for four points: $0^{\circ} \mathrm{N} 0^{\circ} \mathrm{E}$ (1st point), $0^{\circ} \mathrm{N} 45^{\circ} \mathrm{E}$ (2nd point), $4.5^{\circ} \mathrm{N} 0^{\circ} \mathrm{E}$ (3rd point), $9^{\circ} \mathrm{N} 0^{\circ} \mathrm{E}$ (4th point) (see Figure $3(\mathrm{~b})$ ).

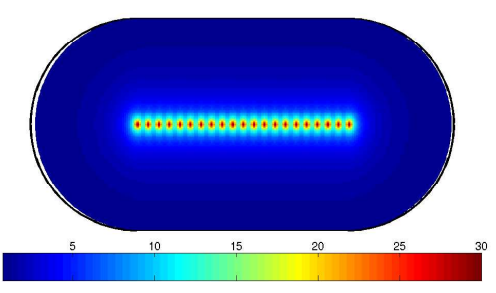

(a) Maximum of the absolute values of the trial function described above

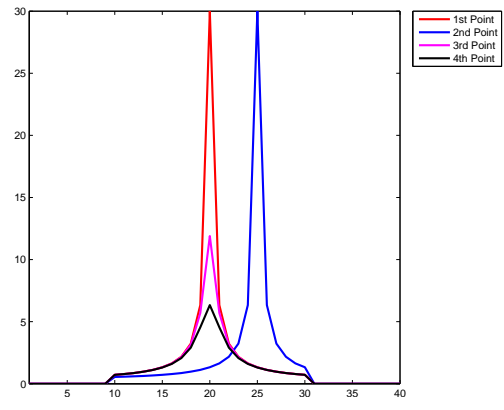

(b) Time dependent courses of the trial function of the four different points given in the text above

Figure 3: Time and space dependent trial function for a moving smoothed peak as described in (3).

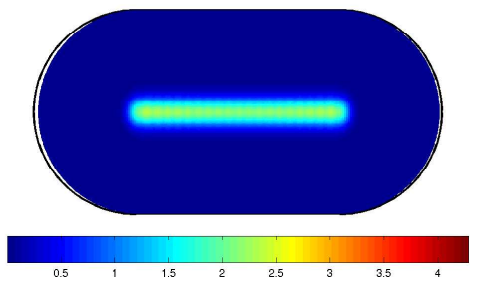

(a) Scale 4

$(\min =0.0120, \max =2.3631)$

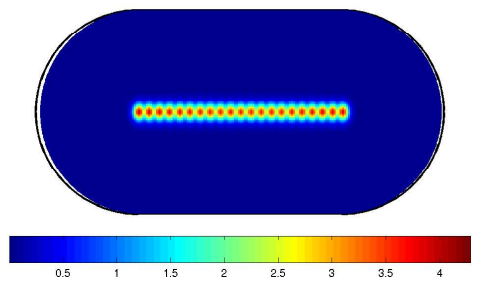

(b) Scale 5

$(\min =0.0053, \max =3.8997)$

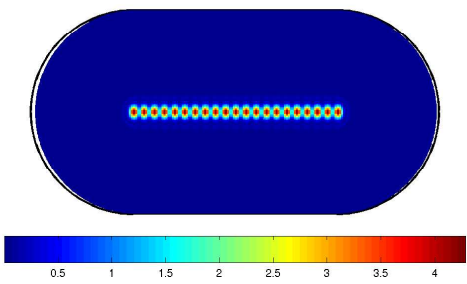

(c) Scale 6

$(\min =0.0026, \max =4.2849)$

Figure 4: Maximum of the absolute values of the pure wavelet coefficients calculated with cubic polynomial wavelet in time and space at different scales based on the data described in the text above.

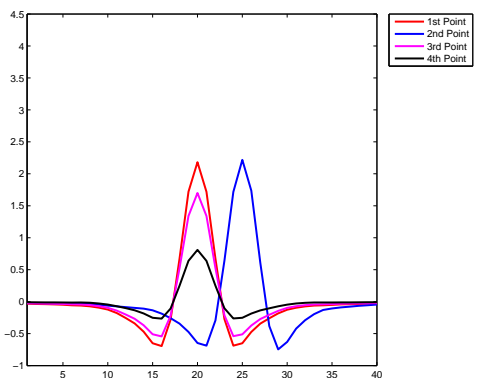

(a) Scale 4

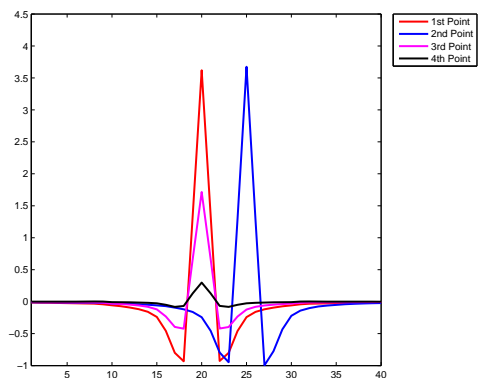

(b) Scale 5

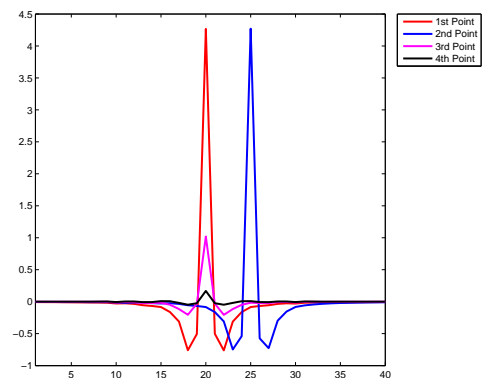

(c) Scale 6

Figure 5: Time dependent courses of the pure wavelet coefficients calculated with cubic polynomial wavelet in time and space at different scales and for the different points based on the data described in the text above. 


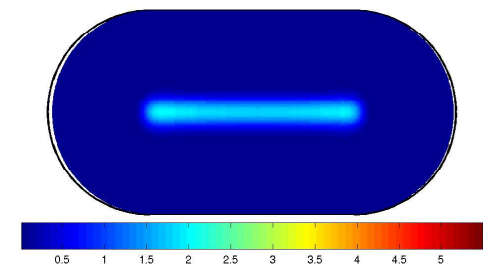

(a) Scale 4

$(\min =0.0369, \max =2.0556)$

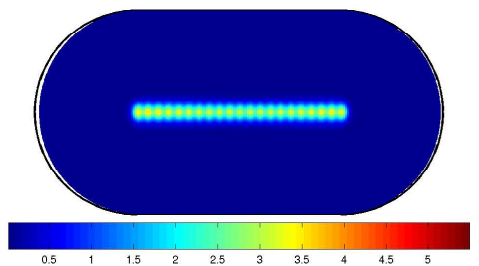

(b) Scale 5

$(\min =0.0194, \max =3.5882)$

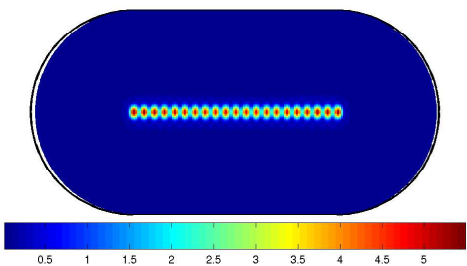

(c) Scale 6

$(\min =0.0111, \max =5.4895)$

Figure 6: Maximum of the absolute values of the first hybrid wavelet coefficients calculated with cubic polynomial wavelet in time and space at different scales based on the data described in the text above.

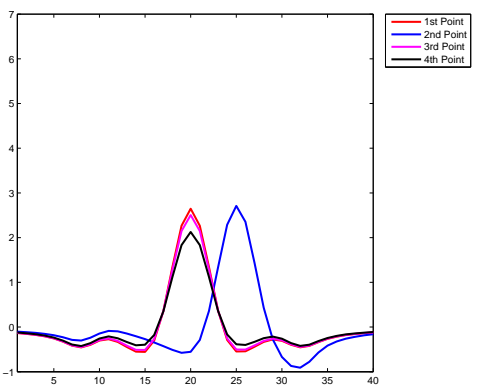

(a) Scale 4

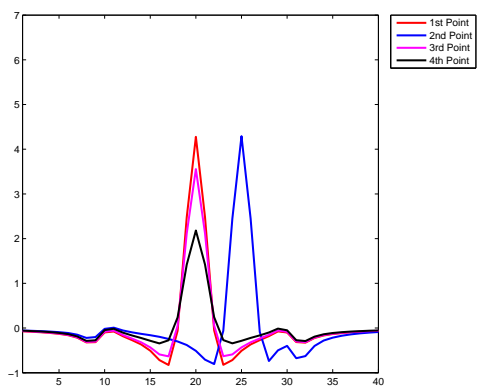

(b) Scale 5

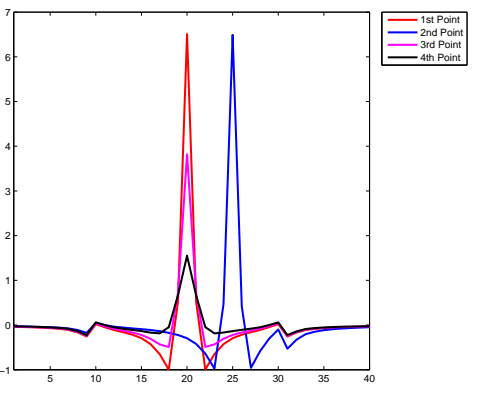

(c) Scale 6

Figure 7: Time dependent courses of the second hybrid wavelet coefficients calculated with cubic polynomial wavelet in time and space at different scales and for the different points based on the data described in the text above.

The results in case of these synthetically generated data meet our expectations. In Figures 4 and 6 you can see the good space localization in case of pure and first hybrid wavelet coefficients and, especially, with increasing scale the several smoothed peaks are more and more localized. The time dependent courses of the pure wavelet coefficients in Figure 5 demonstrate that indeed for each date we find the same structure, i.e., the values of these coefficients are just shifted in time in the same manner as the smoothed peak. The decreasing influence of the different smoothed peaks moving in Northern direction can be well realized. In the time dependent courses of the pure wavelet coefficients the smoothed peak is well detected, but what is missing are the smoothed jumps which we see in Figure 3(b) at date 10 and 30. These smoothed jumps arise due to the fact that from the 10th to the 30th date the small influence of the spatial smoothed peaks at all dates is visible. These jumps do not occur in the time dependent courses of the pure wavelet coefficients because here we localize both in space and time such that the spatial smoothed peak of value 30 dominates the smoothed jump of value about 1 (see Figure $3(\mathrm{~b})$ ). In case of the second hybrid wavelet coefficients these smoothed jumps at date 10 and 30 are well detected because we localize in time and smooth in space (see Figure 7).

\subsection{Earth's Gravitational Data (GRACE)}

In this section we present some results based on Earth's gravitational data sets from the satellite mission GRACE. The data are given as monthly data sets for the time interval from February 
2003 till May 2006.

First, in Section 5.2.1 we start with a short comparison between an original data set and the corresponding wavelet coefficients in order to make clear the efficiency of the time-space multiresolution. After that, we present detailed results by comparing the pure and hybrid wavelet coefficients for different scales.

\subsubsection{Comparison of Original Data and Wavelet Coefficients}

The method introduced in Section 4 provides both temporal and spatial analysis of time series consisting of data sets given on the sphere. In Section 5.1 about synthetically generated data we demonstrated in which way the algorithm filters out certain variations in time and space on the basis of easily structured input data. In this section our aim is to present which temporal and spatial phenomena are found out from real input data as the Earth's gravitational potential.

Because of the results obtained in Section 5.1 we show the changes in the spatial dimension using the first hybrid wavelet coefficients whereas temporal variations are filtered out best by the second hybrid wavelet coefficients. Figure 8 shows the reconstruction of the gravitational potential from the input data, i.e., Fourier coefficients, exemplarily for one month. The first hybrid wavelet coefficients of this selected month are presented in Figure 9 and obviously indicate certain spatial structures.

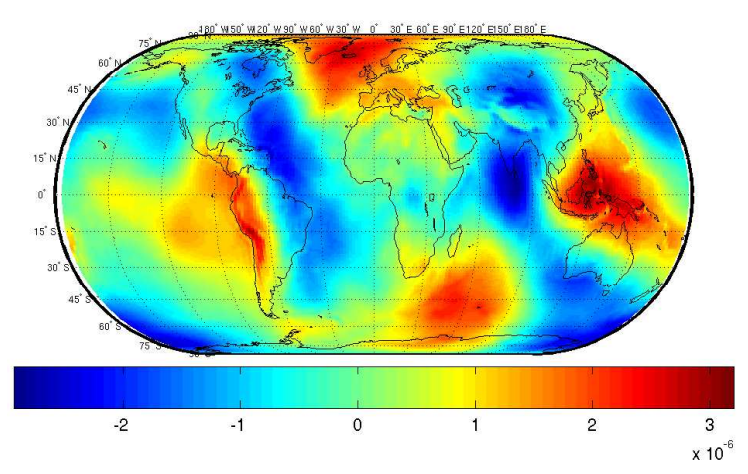

Figure 8: Gravitational potential in May 2005 .

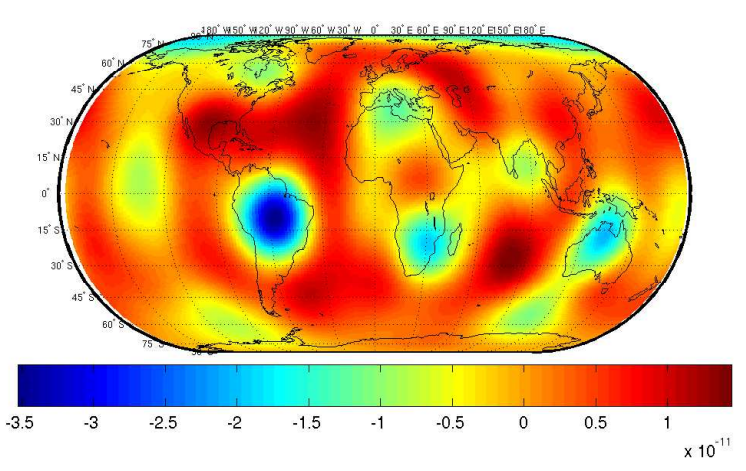

Figure 9: First hybrid wavelet coefficients of May 2005 computed with cubic polynomial wavelet in time and space at scale 3 from a time series of 40 monthly data sets (from February 2003 to May 2006).

In case of the temporal variations we have to fix the position in order to be able to present the time dependent course. On the one hand we choose Manaus located in the Amazonas basin where great seasonal variations appear and on the other hand Kaiserslautern located in a region with moderate temporal changes. Although, at first sight, the time dependent courses of the gravitational potential given in Figure 10 for Manaus and in Figure 12 for Kaiserslautern do not allow to recognize any seasonal structures the time dependent courses of the second hybrid wavelet coefficients (see Figure 11 and Figure 13) show annual oscillations. This structure is more evident in case of Manaus because the absolute values are greater than those in case of Kaiserslautern. But even in Kaiserslautern one can slightly realize different minima in autumn (September/October) and maxima in spring (March/April). 


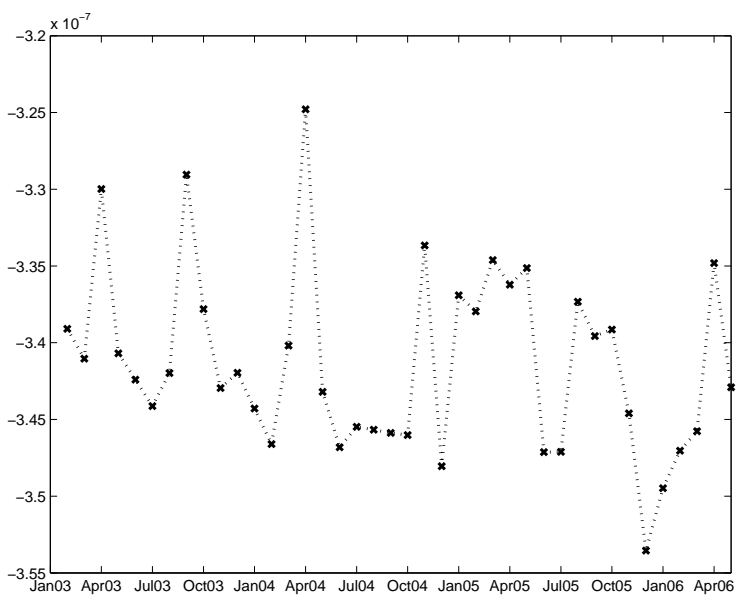

Figure 10: Time dependent course of the gravitational potential from February 2003 to May 2006 in Manaus $\left(3^{\circ} \mathrm{S} 60^{\circ} \mathrm{W}\right)$.

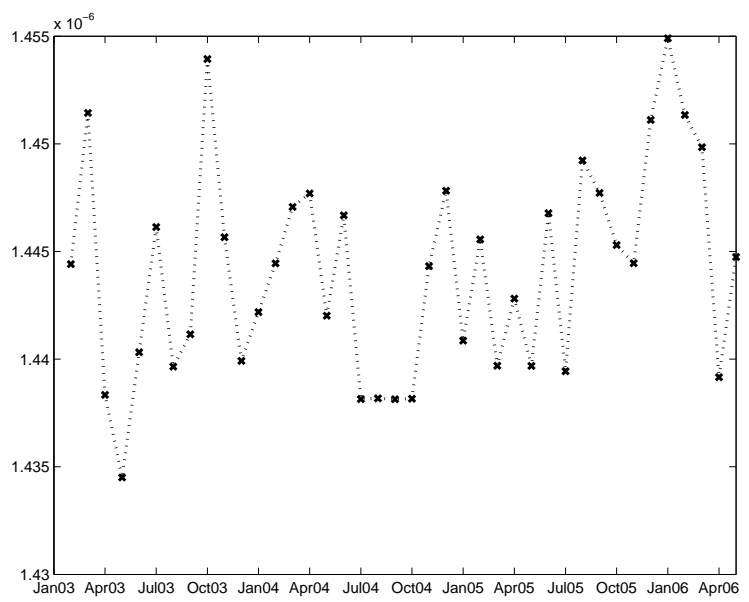

Figure 12: Time dependent course of the gravitational potential from February 2003 to May 2006 in Kaiserslautern $\left(49^{\circ} \mathrm{N} 7^{\circ} \mathrm{O}\right)$.

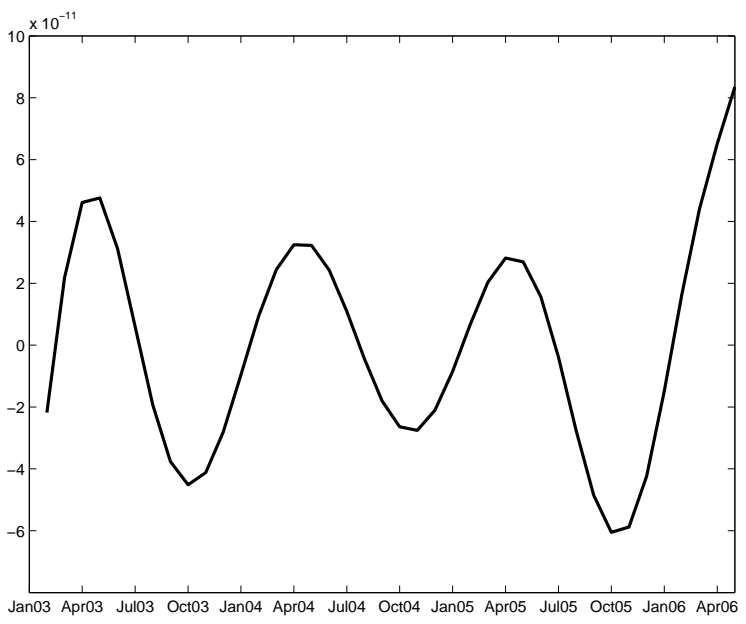

Figure 11: Time dependent course of the second hybrid wavelet coefficients calculated in Manaus with cubic polynomial wavelet in time and space at scale 3 .

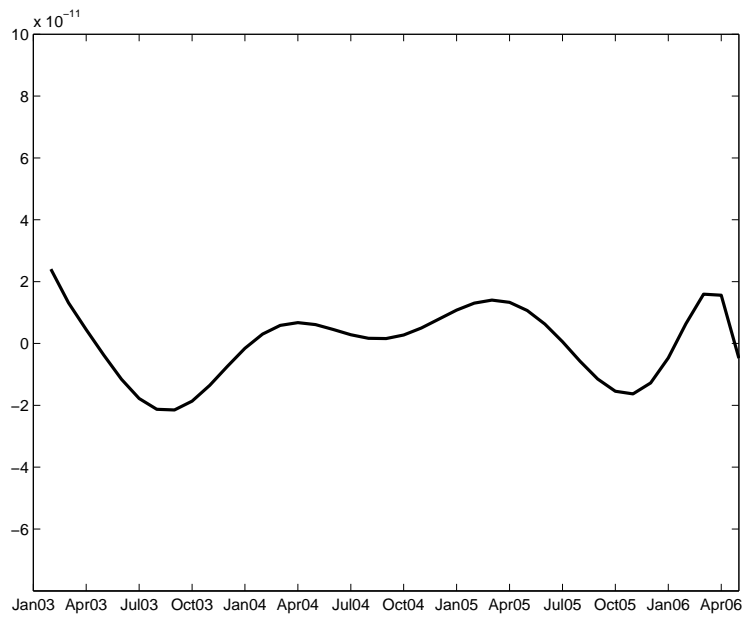

Figure 13: Time dependent course of the second hybrid wavelet coefficients calculated in Kaiserslautern with cubic polynomial wavelet in time and space at scale 3.

\subsubsection{Detailed Results}

After having presented some first comparative results in Section 5.2.1 we are now going to analyze the GRACE data in more detail. On the one hand we compare the wavelet coefficients of different scales and on the other hand we investigate the pure and hybrid wavelet coefficients based on the results of Section 5.1. In case of the spatial analysis we found out that it is reasonable to look at the maximum of the absolute values of the pure and first hybrid wavelet coefficients whereas in case of the temporal analysis the time dependent courses of the pure and 
second hybrid wavelet coefficients for special positions have to be investigated. The results for pure and hybrid wavelets are quite similar but as shown in the example of the moving smoothed peak in Section 5.1 some detail information may only be filtered out using the hybrid wavelet coefficients.

Figure 14 to Figure 16 show the results for the pure wavelet coefficients based on the GRACE data. The plots shown in Figure 14 and Figure 15 make the regions visible where the greatest changes in the potential are (taken over the whole interval of 40 dates), as, e.g., in the Amazonas basin in South America. With increasing scale we can recognize the better space localization but already at scale four shown in Subfigure 15(a) the satellite tracks appear and at scale five they dominate our results. Furthermore, the localization in time is shown in Figure 16, where we recognize that seasonal structures are well extracted. The results in case of hybrid wavelets are shown in Figure 17, Figure 18 and Figure 19. They are quite similar to those of the pure wavelets, but obviously the hybrid wavelet coefficients show more spatial structures. This is due to the fact that the pure wavelet coefficients are dominated by the temporal influence.

For the visualization of the time dependent course we choose exemplarily four different cities, i.e., four different locations in the space domain. We select two points on the Southern and the Northern hemisphere (Manaus, Lilongwe, Dacca and Kaiserslautern). Kaiserlautern is located within a region of moderate seasonal variations in the water balance, whereas the other three cities show the time dependent course in the well-known regions of great changes (Amazonas bassin, region around Lake Malawi and Ganges bassin).

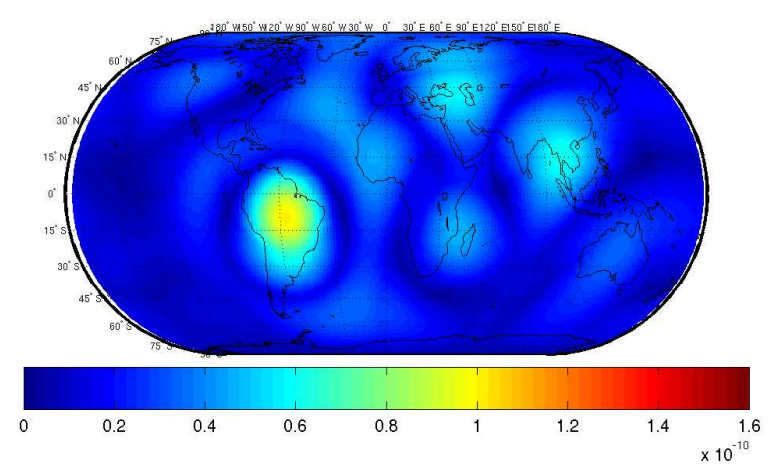

(a) Scale $2\left(\min =0.016 \cdot 10^{-10}, \max =1.011 \cdot 10^{-10}\right)$

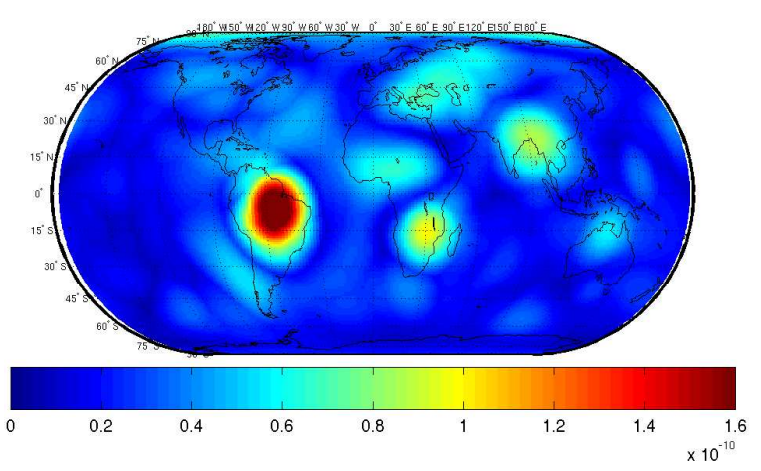

(b) Scale $3\left(\min =0.055 \cdot 10^{-10}, \max =1.976 \cdot 10^{-10}\right)$

Figure 14: Maximum of the absolute values of the pure wavelet coefficients calculated with cubic polynomial wavelet in time and space at different scales based on a time series of 40 monthly data sets (from February 2003 till May 2006). 


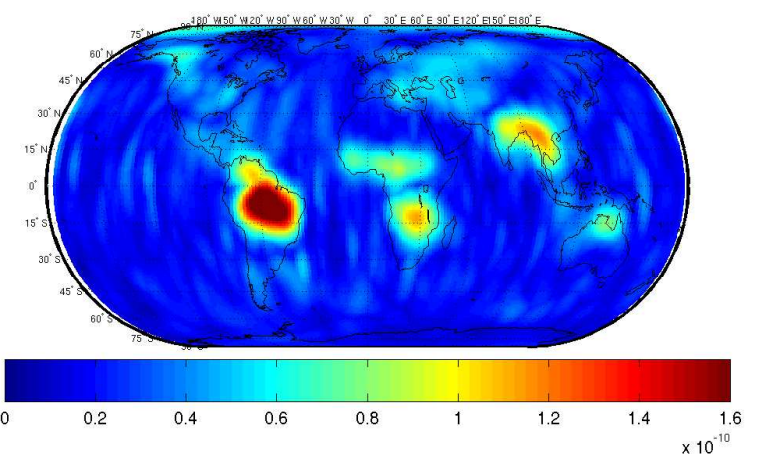

(a) Scale $4\left(\min =0.063 \cdot 10^{-10}, \max =1.981 \cdot 10^{-10}\right)$

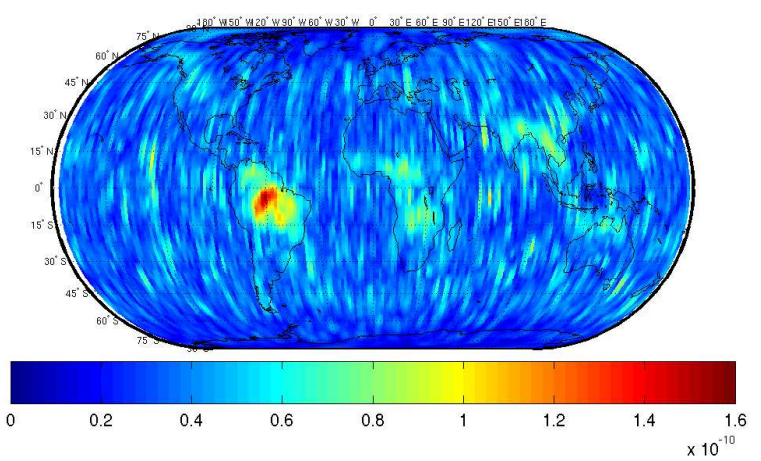

(b) Scale $5\left(\min =0.084 \cdot 10^{-10}, \max =1.563 \cdot 10^{-10}\right)$

Figure 15: Maximum of the absolute values of the pure wavelet coefficients calculated with cubic polynomial wavelet in time and space at different scales based on a time series of 40 monthly data sets (from February 2003 till May 2006).

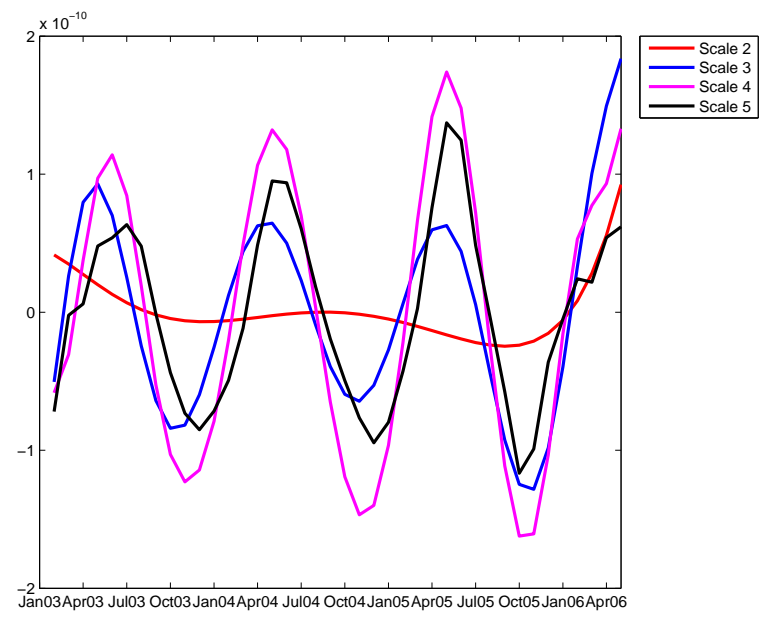

(a) Manaus $\left(3^{\circ} \mathrm{S} 60^{\circ} \mathrm{W}\right)$

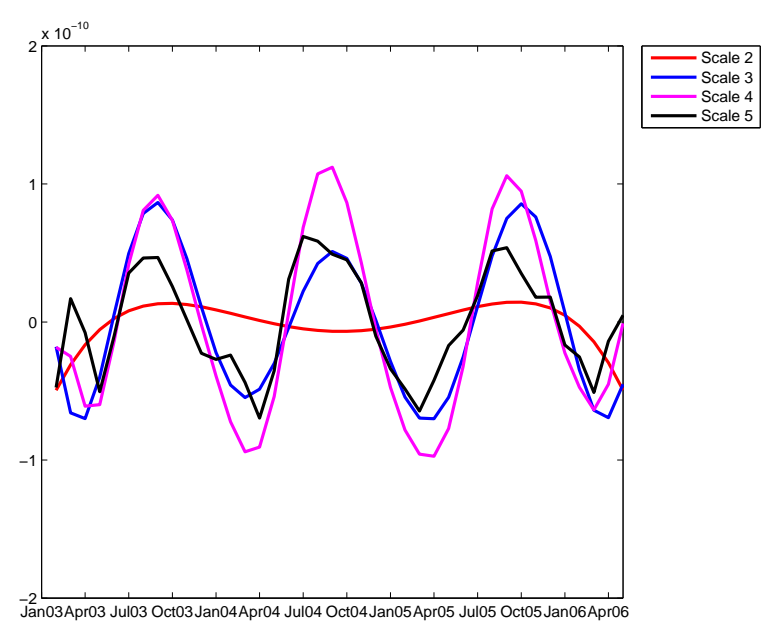

(c) Dacca $\left(23^{\circ} \mathrm{N} 90^{\circ} \mathrm{O}\right)$

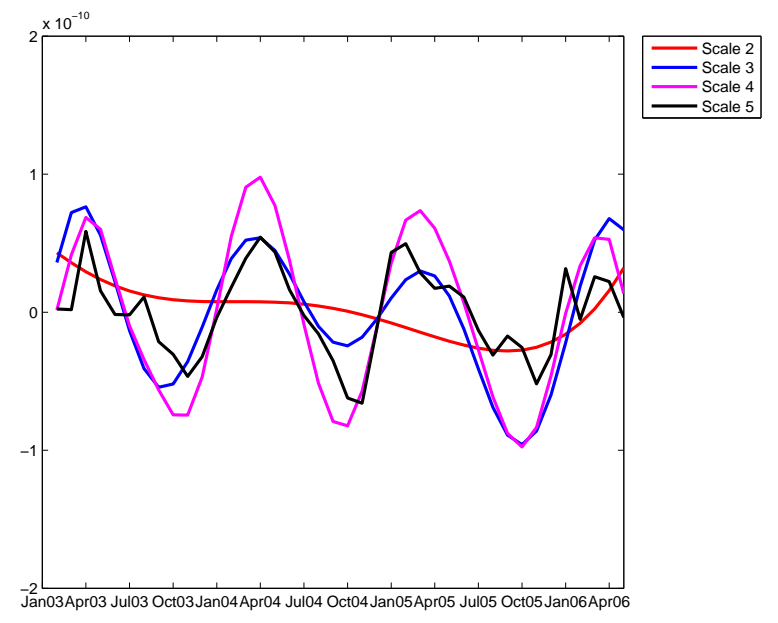

(b) Lilongwe $\left(13^{\circ} \mathrm{S} 33^{\circ} \mathrm{O}\right)$

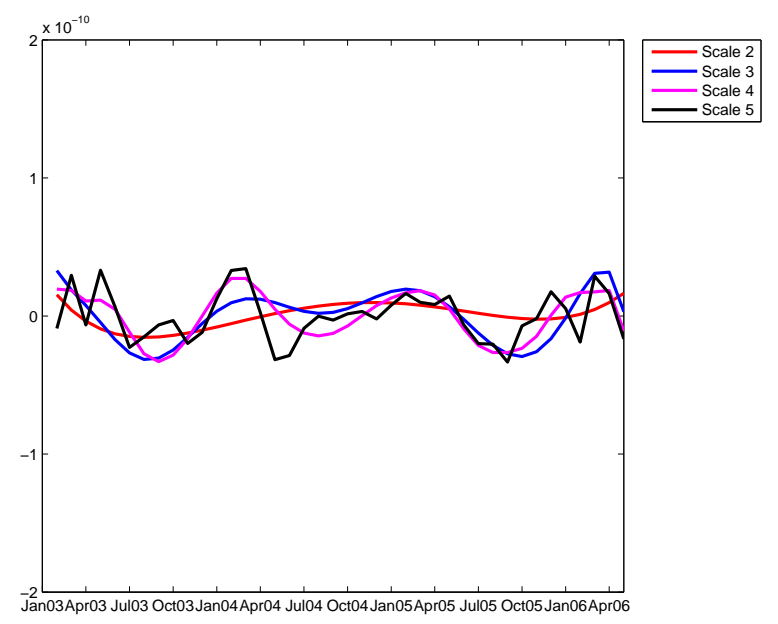

(d) Kaiserslautern $\left(49^{\circ} \mathrm{N} 7^{\circ} \mathrm{O}\right)$

Figure 16: Time dependent courses of the pure wavelet coefficients calculated with cubic polynomial wavelet in time and space at different scales based on a time series of 40 monthly data sets (from February 2003 till May 2006). 


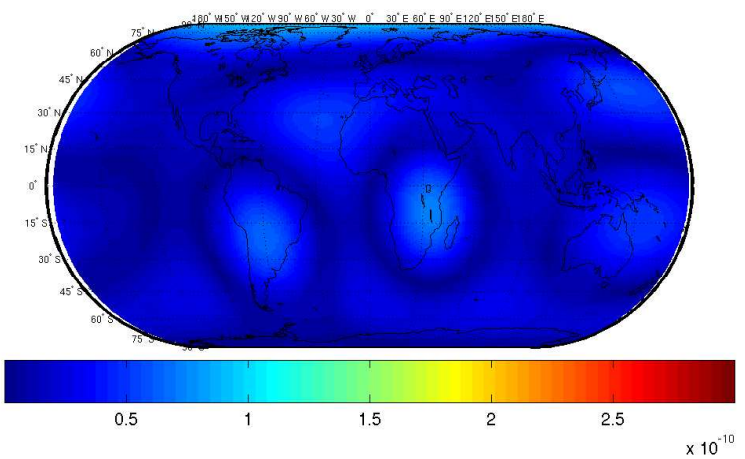

(a) Scale $2\left(\min =0.004 \cdot 10^{-10}, \max =0.893 \cdot 10^{-10}\right)$

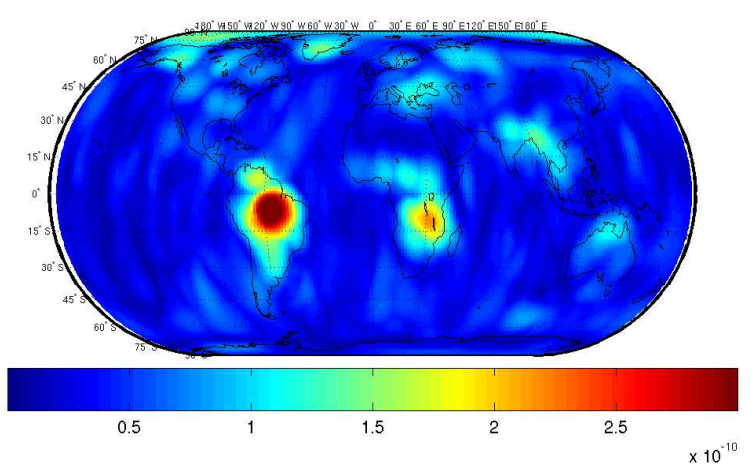

(c) Scale $4\left(\min =0.053 \cdot 10^{-10}, \max =3.652 \cdot 10^{-10}\right)$

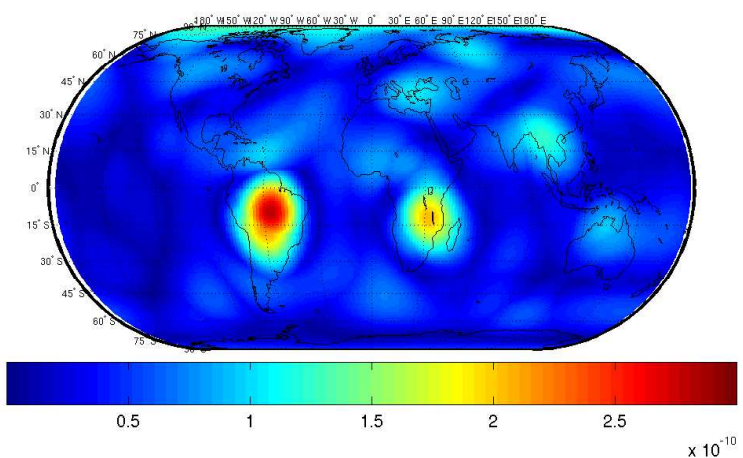

(b) Scale $3\left(\min =0.025 \cdot 10^{-10}, \max =2.810 \cdot 10^{-10}\right)$

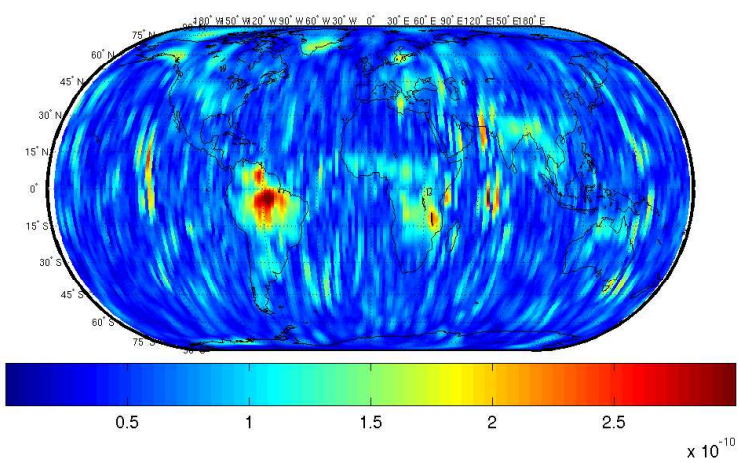

(d) Scale $5\left(\min =0.084 \cdot 10^{-10}, \max =3.278 \cdot 10^{-10}\right)$

Figure 17: Maximum of the absolute values of the first hybrid wavelet coefficients calculated with cubic polynomial wavelet in time and space at different scales based on a time series of 40 monthly data sets (from February 2003 till May 2006).

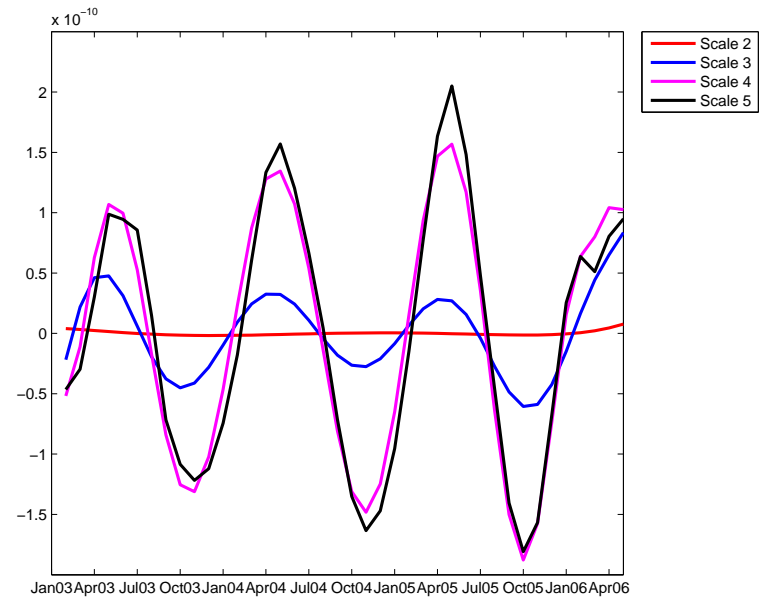

(a) Manaus $\left(3^{\circ} \mathrm{S} 60^{\circ} \mathrm{W}\right)$

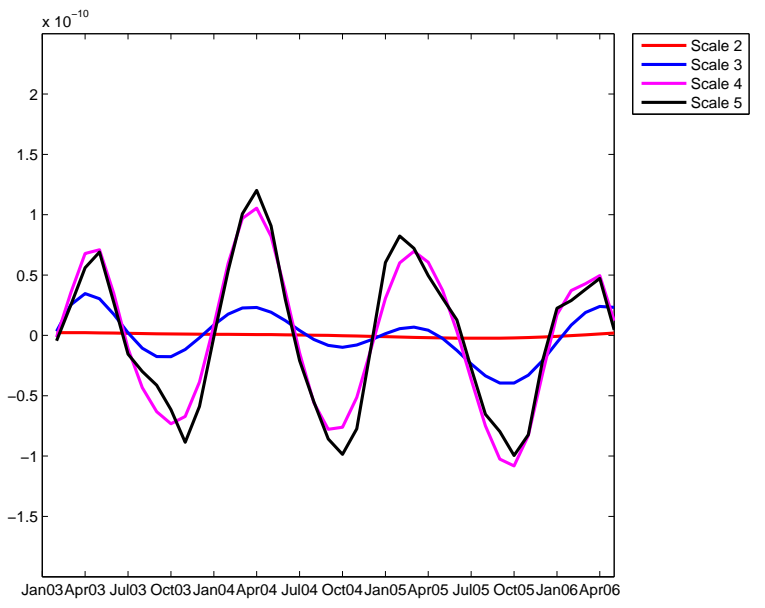

(b) Lilongwe $\left(13^{\circ} \mathrm{S} 33^{\circ} \mathrm{O}\right)$

Figure 18: Time dependent courses of the second hybrid wavelet coefficients calculated with cubic polynomial wavelet in time and space at different scales based on a time series of 40 monthly data sets (from February 2003 till May 2006). 


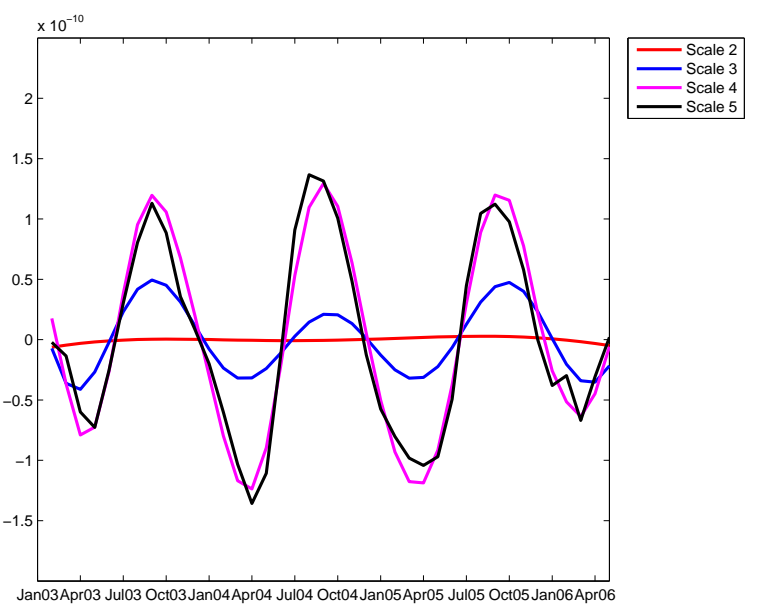

(a) Dacca $\left(23^{\circ} \mathrm{N} 90^{\circ} \mathrm{O}\right)$

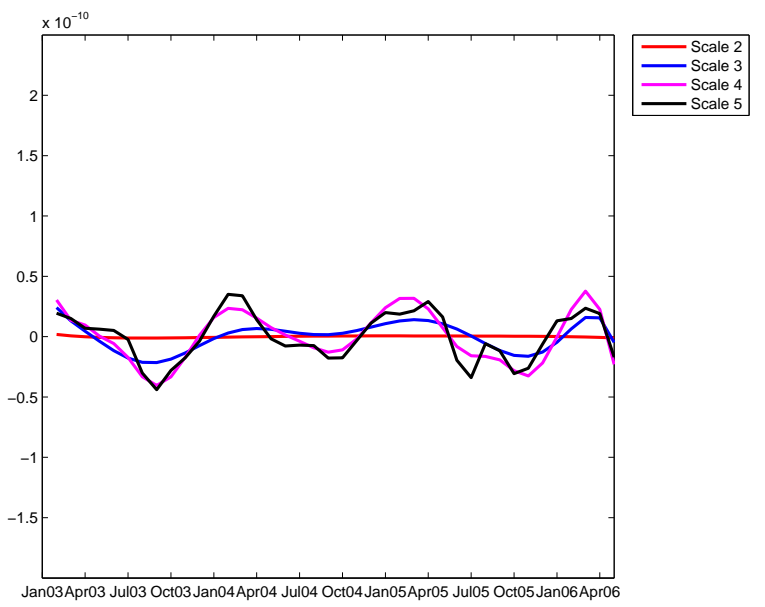

(b) Kaiserslautern $\left(49^{\circ} \mathrm{N} 7^{\circ} \mathrm{O}\right)$

Figure 19: Time dependent courses of the second hybrid wavelet coefficients calculated with cubic polynomial wavelet in time and space at different scales based on a time series of 40 monthly data sets (from February 2003 till May 2006).

In Figure 14, Figure 15 and Figure 17 we see that with increasing scale the regions with great seasonal variations as, e.g., Amazonas basin and Ganges basin, are detected. In scale 5 the influence of the satellite tracks which falsify the results can be seen. In Figure 16, Figure 18 and Figure 19 the time dependent courses of the pure wavelet and second hybrid wavelet coefficients of several cities are plotted. The seasonal variations can be seen best in scales 3 and 4 . Even for Kaiserslautern which is located in a region with moderate variations the course of the wavelet coefficients demonstrates the seasonal course.

\subsection{Hydrology Model WGHM}

We now present some results computed by using WGHM (Water GAP Hydrology Model) data. These data are available as a time series of water columns and are given on a grid of 32400 points. The time series consists of 36 monthly data sets (from January 2003 till December 2005).

As expected the results achieved in case of WGHM data are similar to those presented in Section 5.2.2 for GRACE data. By comparing Figure 20 with Figure 17 on the continents we can obviously recognize the same structures as expected. This is also reflected in the time dependent courses shown in Figure 21, Figure 22, Figure 18 and Figure 19. 


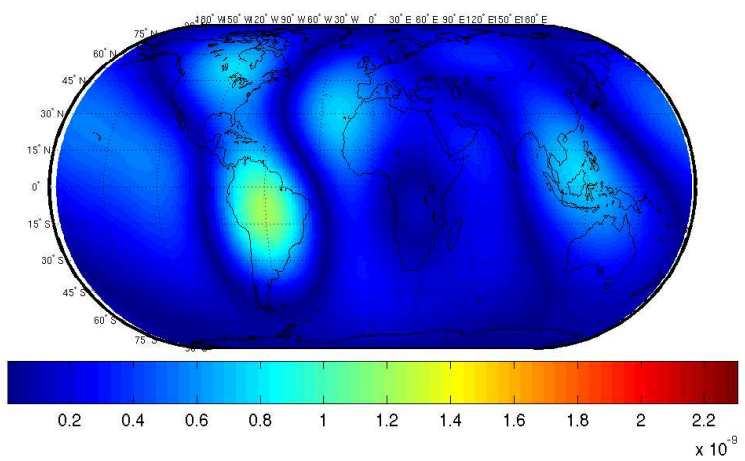

(a) Scale $2\left(\min =0.003 \cdot 10^{-9}, \max =1.230 \cdot 10^{-9}\right)$

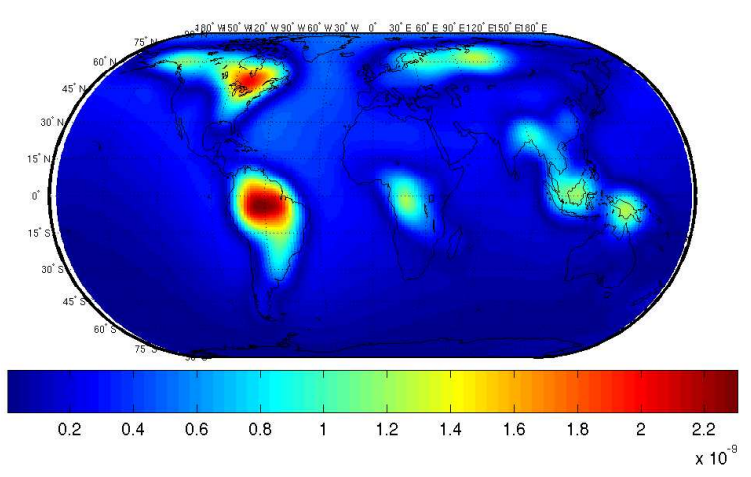

(c) Scale $4\left(\min =0.006 \cdot 10^{-9}, \max =2.304 \cdot 10^{-9}\right)$

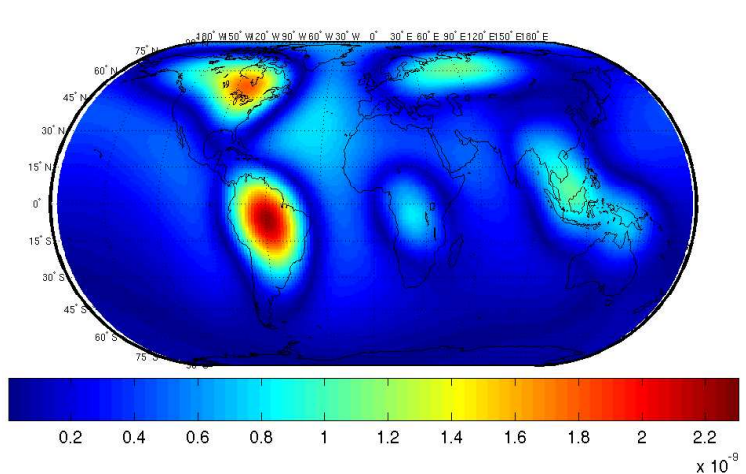

(b) Scale $3\left(\min =0.011 \cdot 10^{-9}, \max =2.224 \cdot 10^{-9}\right)$

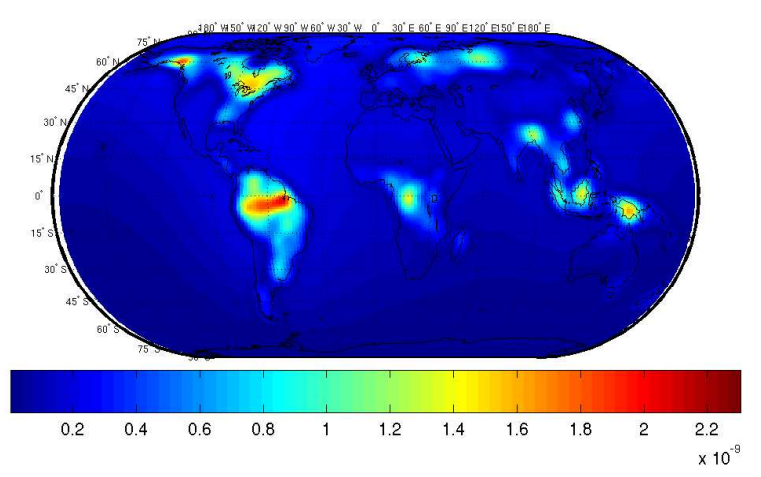

(d) Scale $5\left(\min =0.005 \cdot 10^{-9}, \max =2.045 \cdot 10^{-9}\right)$

Figure 20: Maximum of the absolute values of the first hybrid wavelet coefficients calculated with cubic polynomial wavelet in time and space at different scales based on a time series of 36 monthly data sets (from January 2003 till December 2005).

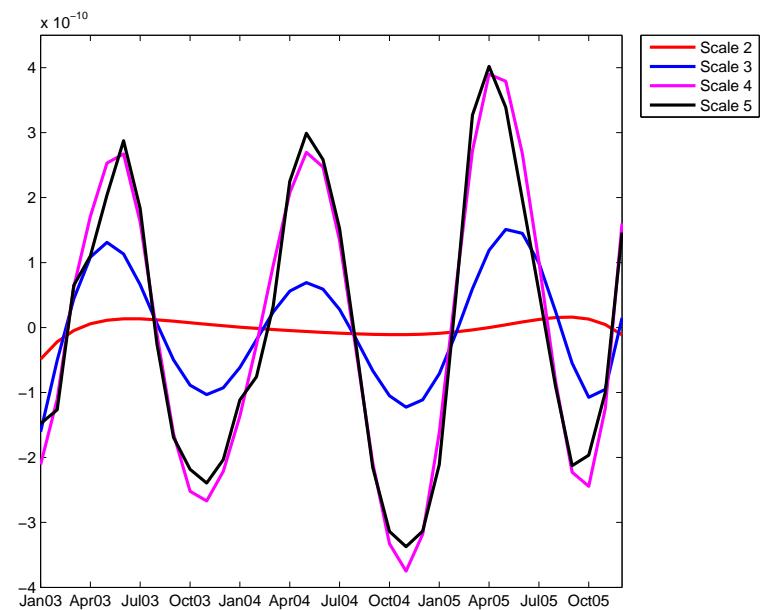

(a) Manaus $\left(3^{\circ} \mathrm{S} 60^{\circ} \mathrm{W}\right)$

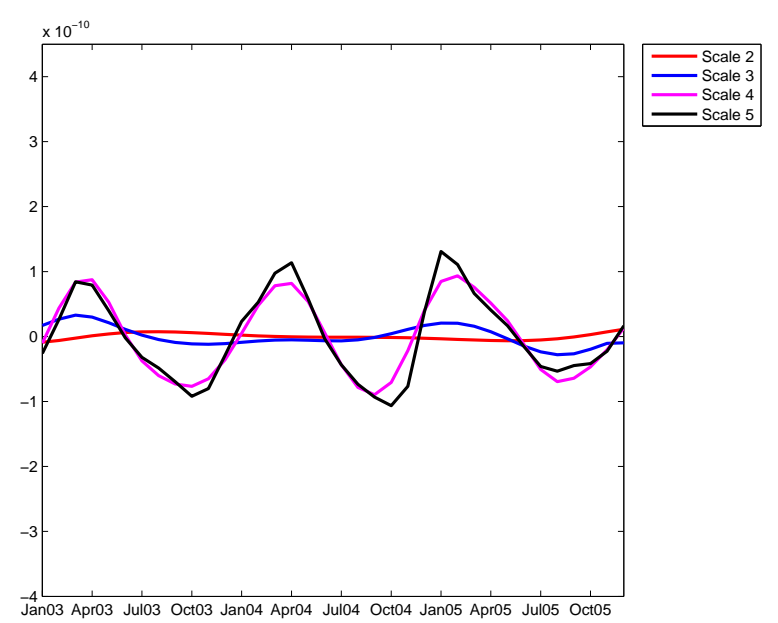

(b) Lilongwe $\left(13^{\circ} \mathrm{S} 33^{\circ} \mathrm{O}\right)$

Figure 21: Time dependent courses of the second hybrid wavelet coefficients calculated with cubic polynomial wavelet in time and space at different scales based on a time series of 36 monthly data sets (from January 2003 till December 2005). 


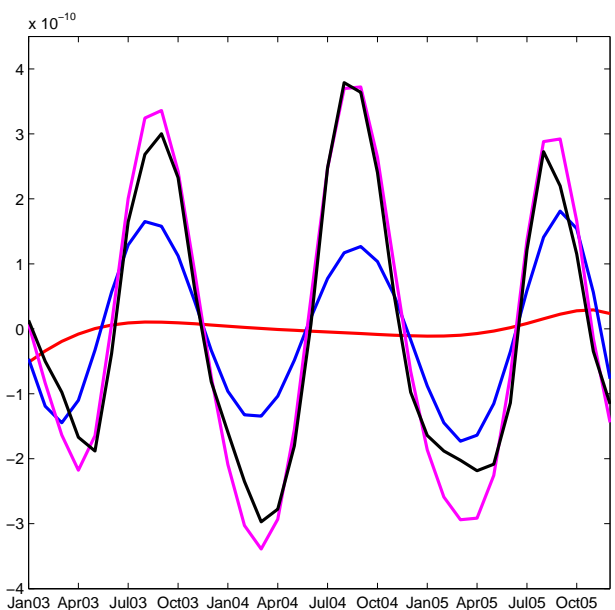

(a) Dacca $\left(23^{\circ} \mathrm{N} 90^{\circ} \mathrm{O}\right)$

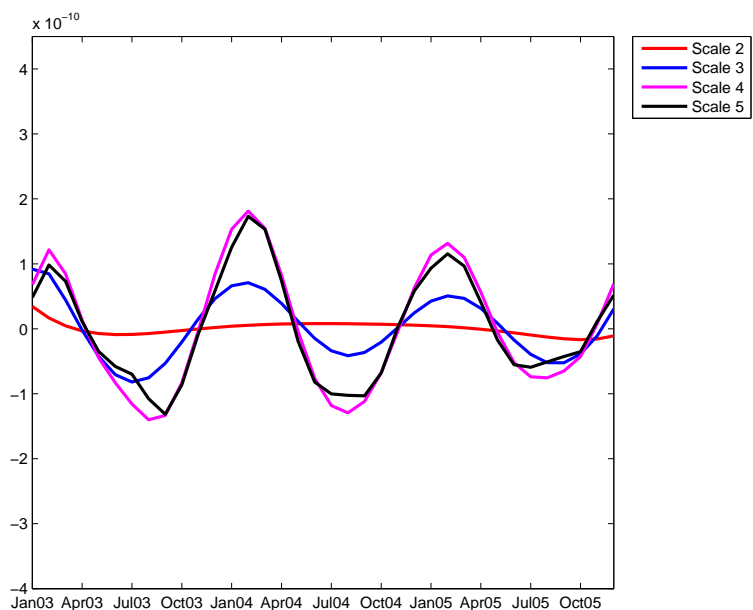

(b) Kaiserslautern $\left(49^{\circ} \mathrm{N} 7^{\circ} \mathrm{O}\right)$

Figure 22: Time dependent courses of the second hybrid wavelet coefficients calculated with cubic polynomial wavelet in time and space at different scales based on a time series of 36 monthly data sets (from January 2003 till December 2005).

\subsection{Atmospheric Short Time Mass Variations}

We now apply the tensor product wavelet analysis on a data set containing 124 short time mass variations in March 2003, i.e., we have one data set every six hours which are given as spherical harmonic coefficients up to degree 100. In March 2003 the short time mass variations have been very small within a region around the equator from $30^{\circ} \mathrm{S}$ to $30^{\circ} \mathrm{N}$, whereas in the remaining region there are great changes which can be seen in Figure 23 and Figure 24.

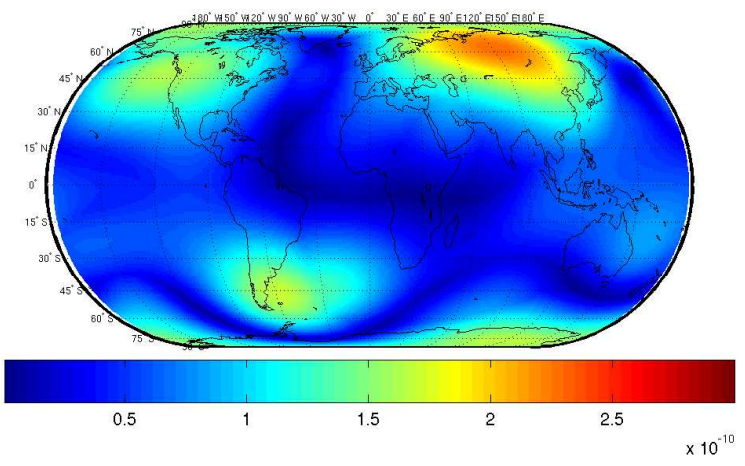

(a) Scale $2\left(\min =0.007 \cdot 10^{-10}, \max =2.293 \cdot 10^{-10}\right)$

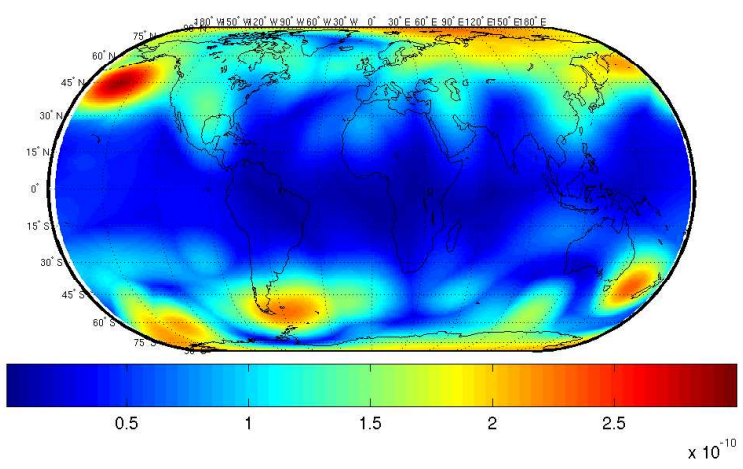

(b) Scale $3\left(\min =0.068 \cdot 10^{-10}, \max =2.867 \cdot 10^{-10}\right)$

Figure 23: Maximum of the absolute values of the first hybrid wavelet coefficients calculated with cubic polynomial wavelet in time and space at different scales based on a time series of 124 data sets from march 2003. 


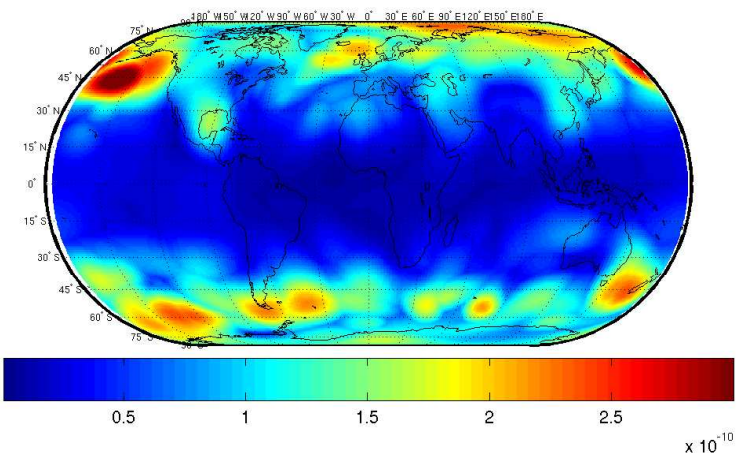

(a) Scale $4\left(\min =0.039 \cdot 10^{-10}, \max =3.353 \cdot 10^{-10}\right)$

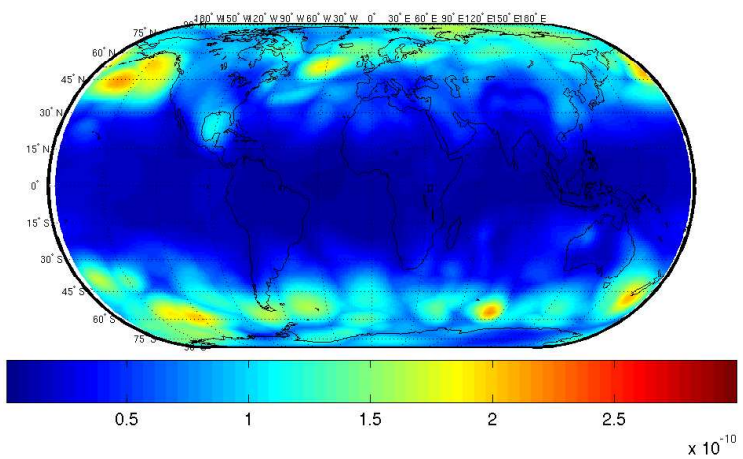

(b) Scale $5\left(\min =0.041 \cdot 10^{-10}, \max =2.183 \cdot 10^{-10}\right)$

Figure 24: Maximum of the absolute values of the first hybrid wavelet coefficients calculated with cubic polynomial wavelet in time and space at different scales based on a time series of 124 data sets from march 2003.

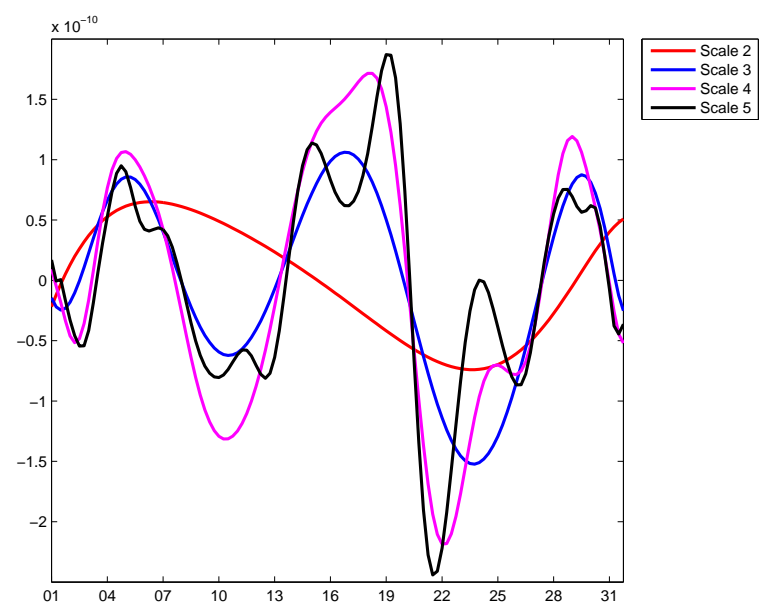

(a) Greenland $\left(70^{\circ} \mathrm{N} 30^{\circ} \mathrm{W}\right)$

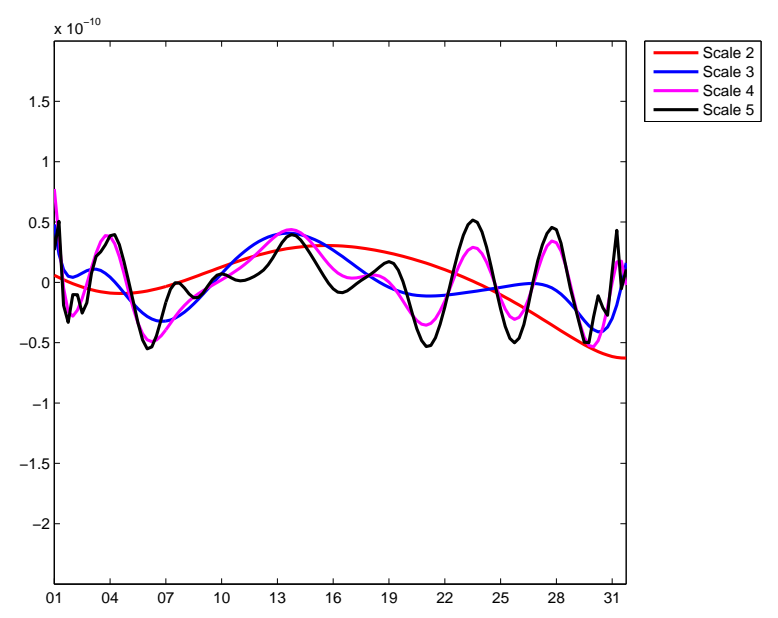

(b) Kampala $\left(0^{\circ} \mathrm{N} 32^{\circ} \mathrm{O}\right)$

Figure 25: Time dependent courses of the second hybrid wavelet coefficients calculated with cubic polynomial wavelet in time and space at different scales based on a time series of 124 data sets from 0:00 a.m. at March 1st 2003 till 6:00 p.m. at March 31th 2003.

As expected no strong changes are detected for the above mentioned region around the equator. In Figure 25 we plot the time dependent courses for one point near the equator (Kampala) and one point in Greenland.

\section{Conclusions}

The aim of this paper is to demonstrate the effetiveness of the time-space multiscale analysis using tensor product wavelets. We apply the algorithm on data sets for gravitational potential and short time mass variations given as spherical harmonic coefficients or water columns on the Earth. Starting from the theory of spherical wavelets introduced by the Geomathematics Group of TU Kaiserslautern tensor product wavelets are built up using the Legendre wavelets in time. This leads to a unified setup of multiresolution in the time space domain because a single 
scale for both time and space suffices. As known from classical multidimensional multiscale analysis we decompose the signal in one smoothed part and three detailed parts. These detailed components are filtered out by use of one pure wavelet and two hybrid wavelets. The algorithm is first demonstrated by means of a synthetically generated example and shows the zooming-in property with increasing scale. Also in case of real data sets the results satisfy our expectations. In the space domain the regions well-known in hydrology for great variations in water balance, as, e.g., the Amazonas bassin, are detected whereas in the time domain the time dependent courses represent seasonal changes which can be explained as hydrological phenomena. Summing up we can state that the time space multiresolution is an efficient algorithm for analyzing temporal and spatial variations.

\section{Acknowledgements}

The authors gratefully acknowledge the support by the Federal Ministry of Education and Research (BMBF) within Geotechnologies Special Programme "Observation System Earth from Space" (03F0424D). We are also much obliged to GFZ Potsdam for providing us with all GRACE and WGHM data. Moreover, we thank the members of the Geomathematics Group of TU Kaiserslautern for valuable discussions.

\section{References}

[1] S. Beth and M. Viell. Uni- und multivariate Legendre-Wavelets und ihre Anwendung zur Bestimmung des Brechungsindexgradienten. In: Progress in Geodetic Science at GW 98. Shaker, W. Freeden, editor, pages 25-33, 1998.

[2] P. Döll, F. Kaspar, and B. Lehner. A global hydrologiecal model for deriving water availability indicators: model tuning and validation. Journal of Hydrology, 270:105-134, 2003.

[3] M.J. Fengler, W. Freeden, A. Kohlhaas, V. Michel, and T. Peters. Wavelet Modelling of Regional and Temporal Variations of the Earth's Gravitational Potential. Schriften zur Funktionalanalysis und Geomathematik, 21, 2005. submitted to Journal of Geodesy.

[4] W. Freeden. Multiscale Modelling of Spaceborne Geodata. Teubner, Stuttgart, Leipzig, 1999.

[5] W. Freeden, T. Gervens, and M. Schreiner. Constructive Approximation on the Sphere With Applications to Geomathematics. Oxford Science Publications, Clarendon, 1998.

[6] W. Freeden and F. Schneider. An Integrated Wavelet Concept of Physical Geodesy. Journal of Geodesy, 72:259-281, 1998.

[7] GRACE Mission. http://www.csr.utexas.edu/grace/.

[8] A.K. Louis, P. Maaß, and A. Rieder. Wavelets: Theorie und Anwendungen. Teubner, Stuttgart, 1998.

[9] Th. Maier. Multiscale Geomagnetic Field Modelling From Satellite Data: Theoretical Aspects and Numerical Applications. PhD thesis, Geomathematics Group, Department of Mathematics, University of Kaiserslautern, 2002.

[10] C. Müller. Spherical Harmonics, volume 17. Springer, Berlin, Heidelberg, New York, 1966. 